\title{
Switching dynamics of surface stabilized ferroelectric liquid crystal cells: Effects of anchoring energy asymmetry
}

\author{
Alexei D. Kiselev* \\ Institute of Physics of National Academy of Sciences of Ukraine, Prospekt Nauki 46, 03028 Kÿ̈, Ukraine \\ Vladimir G. Chigrinov ${ }^{\dagger}$ \\ Hong Kong University of Science and Technology, Clear Water Bay, Kowloon, Hong Kong \\ Eugene P. Pozhidaev \\ P.N. Lebedev Physics Institute of Russian Academy of Sciences, Leninsky Prospect 53, 117924 Moscow, Russia
}

(Received 22 September 2006; published 15 June 2007)

\begin{abstract}
We study both theoretically and experimentally switching dynamics in asymmetric surface stabilized ferroelectric liquid crystal cells where the bounding surfaces are treated differently to produce asymmetry in their anchoring properties. Our electro-optic measurements of the switching voltage thresholds, $V_{+}$and $-V_{-}$, that are determined by the peaks of the reversal polarization current reveal the frequency dependent shift of the hysteresis loop, $V_{+}-V_{-}$. We examine the predictions of the uniform dynamic model with the anchoring energy taken into account. It is found that the asymmetry effects are dominated by the polar contribution to the anchoring energy. Frequency dependence of the voltage thresholds is studied by analyzing the properties of time-periodic solutions to the dynamic equation (cycles). For this purpose, we apply the method linking the cycles and the fixed points of the composition of two parametrized half-period mappings for the approximate model. It is found that the cycles are unstable and can only be formed if the driving frequency is lower than its critical value. The polar anchoring parameter is estimated by making a comparison between the results of modeling and the experimental data for the shift vs frequency curve.
\end{abstract}

DOI: 10.1103/PhysRevE.75.061706

PACS number(s): 61.30.Gd, 61.30.Hn, 77.84.Nh

\section{INTRODUCTION}

As it was shown by Meyer et al. in 1975 [1], the origin of ferroelectric ordering in smectic- $\mathrm{C}^{*}\left(\mathrm{Sm}-\mathrm{C}^{*}\right)$ liquid crystals with the spontaneous polarization normal to the tilt plane is closely related to the reduction of symmetry caused by chirality of the tilted Sm- $\mathrm{C}^{*}$ phase. This phase is thus naturally ferroelectric and the $\mathrm{Sm}-\mathrm{C}^{*}$ liquid crystals are also known as the ferroelectric liquid crystals (FLC) (a detailed description of FLCs can be found, e.g., in a collection of reviews [2] and in more recent monographs [3,4]).

Over the past three decades FLCs have attracted considerable attention not only as a vital issue in the condensed matter physics but also as promising materials for applications in electro-optic switching devices. The first such device was due to Clark and Lagerwall [5]. They studied the electro-optic response of FLC cells confined between two parallel plates subject to homogeneous boundary conditions and made thin enough to suppress the bulk chiral Sm-C* helix. It was found that such cells-the so-called surfacestabilized ferroelectric liquid-crystal (SSFLC) cells-exhibit high-speed, bistable electro-optical switching between orientational states stabilized by surface interactions.

The response of chiral Sm-C $\mathrm{C}^{*}$ liquid crystals to an applied electric field is characterized by fast switching times due to linear coupling between the field and the spontaneous polarization. There is also a threshold voltage necessary for

\footnotetext{
*kiselev@iop.kiev.ua

†eechigr@ust.hk
}

switching to occur and the process of bistable switching is typically accompanied by a hysteresis. The switching times and the threshold voltages may considerably vary depending on the wave form, the amplitude, and the frequency of applied (driving) voltages.

In early studies [6-9], it was found that the competition between elastic, electrostatic, and surface energies may result in different regimes of switching and field induced transitions in FLC cells. Certain regimes such as the high voltage regime can be described using the theoretical approach based on the assumption that the director and polarization fields are spatially homogeneous. This approach provides a number of uniform switching models where the effects of electrostatic and surface interactions are incorporated into an effective potential governing the dynamics of switching. Such models can be readily applied to interpret experimental data.

In particular, the uniform model of switching supplemented with an elasticlike term was examined in [10] and applied to describe experimental behavior of the polarization reversal current in SSFLC cells. Similarly, the uniform theory was employed to determine the rotational viscosity and the anchoring energy strength from the experimental results on the response time measured as a function of pulse voltage in a SSFLC cell [11]. The field-reversal method suggested in [12] to measure the spontaneous polarization, the switching time, the rotational viscosity, and the dc conductivity also relies on the uniform model. (Recent discussions, applications, and generalizations of uniform models can be found, e.g., in [13-16].)

In this paper we are aimed to study the effects of surface anchoring energy in switching dynamics of asymmetric SS- 
FLC cells where nonidentical aligning films impose different boundary conditions at the substrates. The effect of our particular concern is the frequency dependent shift of the hysteresis loop observed in our experiments.

In the theoretical part of the paper, we adapt a systematic approach and examine predictions of the uniform theory where the effects of asymmetry are caused by the polar contribution to the anchoring energy potential. The voltage thresholds are studied in relation to the driving frequency by using the method suggested to explore the properties of timeperiodic solutions to the dynamic equation representing periodic regimes of switching. In the theory of dynamical systems such solutions are variously known as the periodic orbits or the cycles $[17,18]$.

The layout of the paper is as follows. In Sec. II we derive the effective potential and formulate the model. The simplified case with the anchoring energy neglected is discussed so as to clarify the assumptions taken to obtain frequency dependent threshold voltages.

Analysis of the switching dynamics is performed in Sec. III. We present the approach that uses the parametrized halfperiod mappings for the approximate model to study the time-periodic solutions of the dynamic equation as the fixed points of the composition of two half-period mappings. In the case of square wave voltages the method is applied to derive analytical relations for the conditions of complete switching, the switching times, and the critical frequency bounding the region of cycles from above. We show that, for sinusoidal and triangular wave forms, behavior of the halfperiod mappings is similar to that for the square wave voltage and the results remain qualitatively unchanged.

Experimental details and the results of electro-optic measurements for the switching voltage thresholds are given in Sec. IV. The experimental data and the theoretical results of Sec. III are used to model the process of switching and to estimate the polar anchoring parameter. Finally, in Sec. V we present our results and make some concluding remarks.

\section{MODEL}

In this section we introduce necessary notations and describe the model that takes into account the effects due to aligning films. The equation of motion governing switching dynamics in asymmetric SSFLC cells is derived and the asymmetry induced polar contribution to the anchoring energy is found to play an important part in the problem. We also discuss applying the simplified model to describe the frequency dependence of the switching voltage thresholds.

\section{A. Free energy}

We consider the FLC cell with a planar arrangement of planar smectic layers usually referred to as the bookshelf geometry. This geometry is schematically presented in Fig. 1. It is characterized by the director field

$$
\hat{\mathbf{n}}=\hat{\mathbf{z}} \cos \theta+\sin \theta(\hat{\mathbf{x}} \cos \phi+\hat{\mathbf{y}} \sin \phi), \quad \hat{\mathbf{k}}=\hat{\mathbf{y}},
$$

where $\theta$ is the molecular cone angle, $\phi$ is the azimuthal angle around the smectic cone, $\hat{\mathbf{k}}$ is the outward (inward) normal to

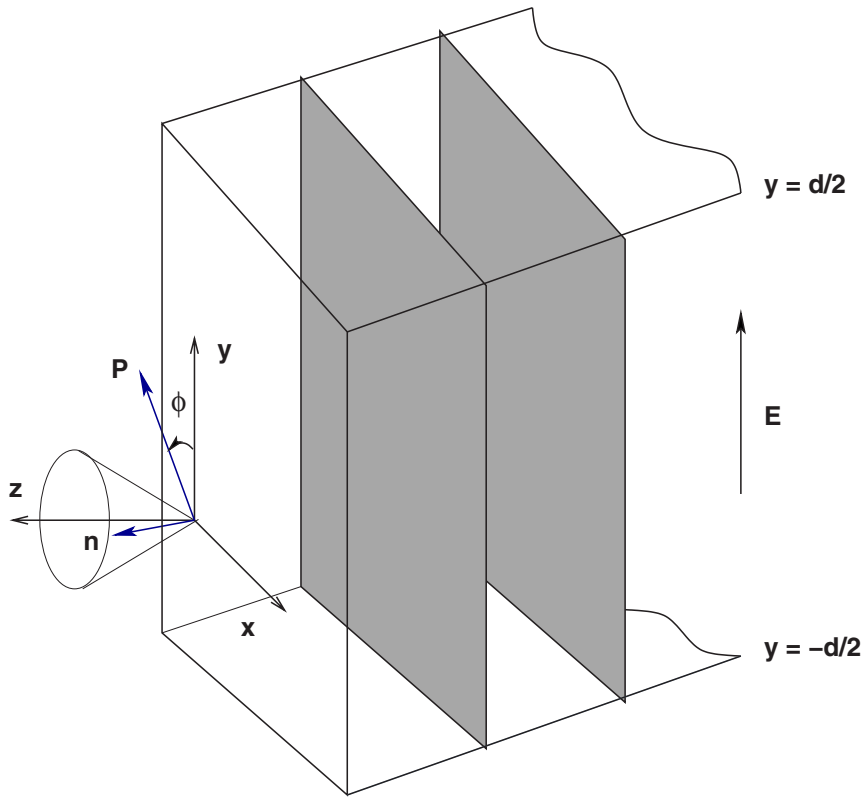

FIG. 1. (Color online) Schematic representation of bookshelf geometry illustrating the planar arrangement of smectic layers. The polarization vector lies in the $x-y$ plane and $\phi$ is the azimuthal angle around the smectic cone. External electric field is directed along the $y$-axis normal to the boundary surfaces, $y=-d / 2$ and $y$ $=d / 2$.

the upper (lower) substrate of FLC cell, $y=d / 2(y=-d / 2)$, and $d$ is the cell thickness.

The vector of the polarization, $\mathbf{P}$, and the electric field inside the cell, $\mathbf{E}$, are given by

$$
\mathbf{P}=P_{\mathrm{S}} \hat{\mathbf{p}}, \quad \hat{\mathbf{p}}=\hat{\mathbf{y}} \cos \phi-\hat{\mathbf{x}} \sin \phi, \quad \mathbf{E}=E \hat{\mathbf{y}},
$$

where $P_{\mathrm{S}}$ is the spontaneous ferroelectric polarization. The external driving voltage

$$
V(t)=V_{\mathrm{m}} v(t)
$$

is characterized by the amplitude $V_{\mathrm{m}}$, the frequency $f=1 / T$ ( $T$ is the period), and the $T$-periodic function of time $v(t)$ describing the wave form of the applied voltage.

For sinusoidal (sine-wave) driving voltages, the waveform function $v(t)$ is

$$
v_{\text {sine }}(t)=\sin (\omega t), \quad \omega=2 \pi / T,
$$

whereas $T$-periodic continuations of the functions $v_{\text {triang }}(t)$ and $v_{\text {squar }}(t)$ :

$$
\begin{gathered}
v_{\text {triang }}(t)= \begin{cases}4 t / T, & 0 \leqslant t / T \leqslant 1 / 4, \\
2-4 t / T, & 1 / 4 \leqslant t / T \leqslant 3 / 4, \\
4 t / T-4, & 3 / 4 \leqslant t / T \leqslant 1,\end{cases} \\
v_{\text {squar }}(t)= \begin{cases}1, & 0 \leqslant t / T<1 / 2, \\
-1, & 1 / 2 \leqslant t / T<1,\end{cases}
\end{gathered}
$$

give the wave-form functions for the triangular and the square-wave forms, respectively.

Similar to nematic liquid crystals (NLC), when a FLC layer is brought into contact with aligning substrates, the 
energy of the FLC molecules in the interfacial layer and thus the surface tension will be orientationally dependent. The anisotropic part of the surface tension-the so-called anchoring energy - gives rise to the phenomenon known as anchoring, i.e., surface induced alignment of the FLC director along the vector of preferential orientation referred to as the easy axis.

The phenomenological expression for the anchoring energy can be written as a linear combination of the invariants constructed from the surface normal, $\hat{\mathbf{k}}$, the FLC director, $\hat{\mathbf{n}}$, and the unit polarization vector $\hat{\mathbf{p}}$. For FLC cells, this gives the anchoring energy expressed in terms of the scalar products $(\hat{\mathbf{n}} \cdot \hat{\mathbf{k}})$ and $(\hat{\mathbf{p}} \cdot \hat{\mathbf{k}})$ as follows:

$$
W_{\text {anch }}=\sum_{\nu= \pm 1}\left[\frac{W_{\nu}}{2}(\hat{\mathbf{n}} \cdot \hat{\mathbf{k}})^{2}+\nu W_{\nu}^{(P)}(\hat{\mathbf{p}} \cdot \hat{\mathbf{k}})\right]_{y=\nu d / 2},
$$

where $W_{+}\left(W_{-}\right)$is the strength of nonpolar anchoring at the upper (lower) substrate; similarly, $W_{+}^{(P)}$ and $W_{-}^{(P)}$ is the polar anchoring strength at the upper and lower substrate, respectively.

The first term in square brackets on the right-hand side of Eq. (7) represents the Rapini-Papoular surface potential [19] preserving equivalence between $\hat{\mathbf{n}}$ and $-\hat{\mathbf{n}}$. This equivalence, however, can be broken due to effects of polar ordering in the interfacial layer $[20,21]$.

As opposed to the case of nematic liquid crystals, where the effects of surface induced polarity are mainly caused by the quadrupole-dipole interaction [22,23], it might be expected that in FLCs the dominating factor is the electrostatic interaction of the spontaneous polarization $\mathbf{P}$ with the surface charges or dipoles. So, the polar anchoring term on the right hand side of Eq. (7) is taken to be proportional to $(\hat{\mathbf{p}} \cdot \mathbf{k})$. This introduces dependence on the polarity of the polarization resulting from the ferroelectric polar surface interaction $[8,24,25]$. There is also the elastic term describing the effect of spontaneous bend which is of the divergence form and can be considered as an additional contribution to the polar term $[25,26]$.

When the director field (1) is spatially homogeneous, the anchoring energy (7) takes the simplified form

$$
W_{\text {anch }}=\frac{W}{2} \sin ^{2} \phi-W_{P} \cos \phi,
$$

where $W=\left(W_{-}+W_{+}\right) \sin ^{2} \theta$ and $W_{P}=W_{-}^{(P)}-W_{+}^{(P)}$. From Eq. (8) it is seen that, for symmetric cells with $W_{-}^{(P)}=W_{+}^{(P)}$, the polar terms add to zero. In asymmetric FLC cells, the polar anchoring parameter, $w_{p}=W_{P} / W$, is generally nonvanishing.

In Fig. 2, the potential (8) is plotted in relation to the azimuthal angle $\phi$. It is illustrated that there are two local minima: $\phi=0$ [the up state with $p \equiv(\hat{\mathbf{p}} \cdot \mathbf{k})=\cos \phi=+1$ ] and $\phi=\pi$ (the down state with $p=-1$ ). At $w_{p} \neq 0$, the minima are not energetically equivalent and one of the minima represents the metastable state. In addition, it is not difficult to obtain the relation

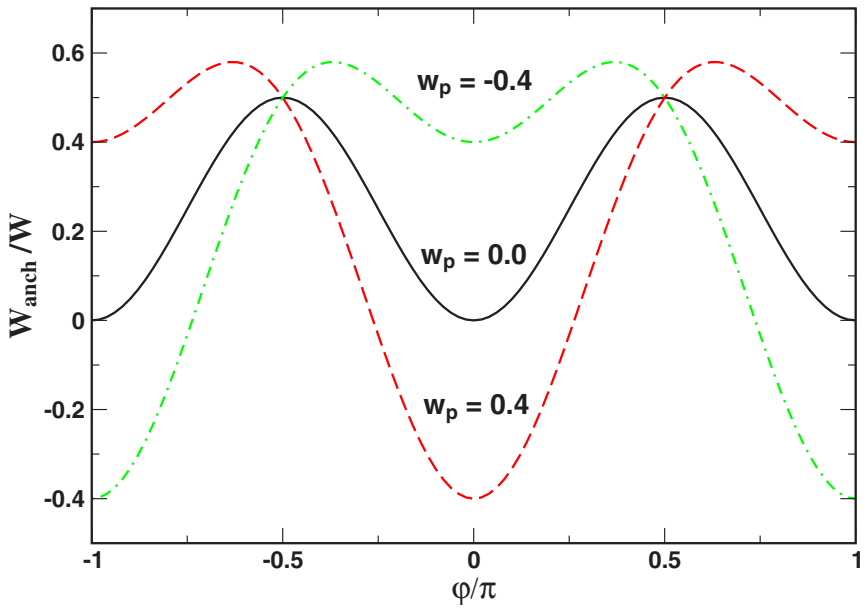

FIG. 2. (Color online) Anchoring energy as a function of the azimuthal angle $\phi$ at various values of the polar anchoring parameter $w_{p} \equiv W_{p} / W$

$$
\left|w_{p}\right|=\left|W_{P} / W\right|<1
$$

as the condition for the configurations with $\phi=0$ and $\phi=\pi$ to be metastable. If the metastability condition (9) is broken the anchoring potential has only one minimum. Similar results were previously reported in Refs. [15,27] and the effects of asymmetry induced by the polar anchoring in switching dynamics will be our primary concern. Throughout the paper we shall assume that the up and down states are both metastable in the absence of external fields and the condition (9) is satisfied.

In general, the polar anchoring energy is known to be of considerable importance in the understanding of static and dynamical behavior of chiral smectics in confined geometries. For example, it may lead to the discontinuous Fréedericksz transitions $[7,8]$ and is found to give rise to the surface electroclinic effect $[28,29]$. The magnitude of the polar anchoring strength was also estimated from the experimental data on the static dielectric susceptibility, the voltage coercivity, and the relaxation time [30].

In an antiferroelectric liquid crystal cell, where the polar anchoring induces ferroelectric ordering close to the bounding surfaces, it may result in a coexistence of multiple zerovoltage ground states [31]. For SSFLC cells, the effects of the polar anchoring energy were recently studied in the context of theoretical investigations into the so-called "thresholdless" or "V-shaped" switching mode of the optical response to an applied voltage $[27,32,33]$. In particular, it was found that monostable structures exhibiting the V-shaped switching can be formed as a result of the polarization charge self-interaction accompanied by the effects of the insulating alignment layers.

So, we briefly discuss a simple double-layer model where FLC is assumed to be sandwiched between two nonidentical insulating layers. The starting point is the standard expression for the electrostatic part of the FLC free energy per unit area 


$$
F_{E} / A=\int_{-d / 2}^{d / 2} f_{E} d y, \quad f_{E}=-\varepsilon_{0} \varepsilon_{y y} E^{2} / 2-P_{y} E,
$$

where $A$ is the area of the substrates, $\varepsilon_{0}=8.854$ $\times 10^{-12} \mathrm{~F} \mathrm{~m}^{-1}$ is the permittivity of free space, and $\varepsilon_{i j}$ $=\varepsilon_{\perp} \delta_{i j}+\left(\varepsilon_{\|}-\varepsilon_{\perp}\right) n_{i} n_{j}$ is the FLC dielectric tensor.

Then we neglect the conductivity of FLC and use the boundary conditions for the normal component of the electric displacement field, $D_{y}$, to obtain the relations

$$
\varepsilon_{0} \varepsilon_{y y} E+P_{y}=\varepsilon_{0} \varepsilon_{1} E_{1}=\varepsilon_{0} \varepsilon_{2} E_{2}=D_{y},
$$

where $\varepsilon_{1}\left(\varepsilon_{2}\right)$ is the dielectric constant of the lower (upper) insulating layer and $E_{i}$ is the electric field inside the layers. The voltage applied across the cell is given by

$$
V \equiv V\left(-d / 2-d_{1}\right)-V\left(d / 2+d_{2}\right)=E_{1} d_{1}+E_{2} d_{2}+\int_{-d / 2}^{d / 2} E d y,
$$

where $d_{i}$ is the thickness of the layers.

We can now use Eq. (11) to eliminate the electric fields, $E_{1}$ and $E_{2}$, from the expression (12) and relate the electric displacement field to the external electric field, $E_{0}$, as follows:

$$
V / d \equiv E_{0}=\eta D_{y} / \varepsilon_{0}+\langle E\rangle, \quad \eta=\left(d_{1} / \varepsilon_{1}+d_{2} / \varepsilon_{2}\right) / d,
$$

where $\langle\cdots\rangle=d^{-1} \int_{-d / 2}^{d / 2} \cdots d y$. This result can now be combined with the relation (11) to yield the expression for the electric field inside the FLC layer

$$
E=\frac{\varepsilon_{0} E_{0}-\eta P_{y}+\left\langle P_{y} \varepsilon_{y y}^{-1}\right\rangle-P_{y}\left\langle\varepsilon_{y y}^{-1}\right\rangle}{\varepsilon_{0} \varepsilon_{y y}\left[\left\langle\varepsilon_{y y}^{-1}\right\rangle+\eta\right]} .
$$

For a uniform director distribution, Eq. (14) can be simplified giving the relation

$$
E=\frac{E_{0}-\eta P_{y} / \varepsilon_{0}}{1+\varepsilon_{y y} \eta}
$$

recently obtained in Ref. [34] for the case of identical substrates. Evidently, this relation implies that the electric field inside the FLC layer, $E$, deviates from the applied electric field, $E_{0}$, due to the presence of the aligning substrates. According to Refs. $[33,35]$, this effect plays an important part in describing the V-shaped switching mode.

Alternatively, Eq. (15) can be used to introduce the effective electrostatic free energy

$$
2 f_{\text {eff }}=-\left(1+\eta \varepsilon_{y y}\right)^{-1}\left[\varepsilon_{0} \varepsilon_{y y} E_{0}^{2}+2 E_{0} P_{y}-\eta P_{y}^{2} / \varepsilon_{0}\right],
$$

which is defined so as to meet the condition

$$
-\frac{\partial f_{E}(\phi, E)}{\partial \phi}=\frac{\partial \varepsilon_{y y}}{\partial \phi} \varepsilon_{0} E^{2} / 2+\frac{\partial P_{y}}{\partial \phi} E=-\frac{\partial f_{\mathrm{eff}}\left(\phi, E_{0}\right)}{\partial \phi}
$$

expressing equivalence of the torques computed from the free energy (10) and the effective energy (16). The last term in the square brackets on the right-hand side of Eq. (16) represents the energy of self-interacting polarization charges.

Now we neglect the dielectric anisotropy of FLC, $\varepsilon_{y y}$ $\approx \varepsilon_{\mathrm{FLC}}=\varepsilon_{\perp}$, and consider the energy

$$
F \equiv f d=f_{\text {eff }} d+W_{\text {anch }}
$$

defined as the sum of the effective electrostatic and anchoring energies. After substituting Eqs. (8) and (16) into the energy (18), it can be seen that there are two effects caused by the aligning layers: (a) reduction of the applied electric field: $E_{0} \rightarrow\left[1+\eta \varepsilon_{\mathrm{FLC}}\right]^{-1} E_{0}$, and (b) renormalization of the nonpolar anchoring strength induced by the polarization selfinteraction: $W \rightarrow W-\eta\left[1+\eta \varepsilon_{\mathrm{FLC}}\right]^{-1} \varepsilon_{0}^{-1} P_{\mathrm{S}}^{2}$. The latter is the effect that may break the metastability condition (9) and give rise to monostable structures exhibiting the thresholdless switching [27,32]. Since such a possibility as well as the $\mathrm{V}$-shaped switching is beyond the scope of this paper, we shall use the energy (18) without changing notations for the renormalized values of $E_{0}$ and $W$.

The simple model of switching dynamics in FLC cells can be formulated in terms of the energy (18) which enters the dynamic equation for the azimuthal angle

$$
\gamma \frac{\partial \phi}{\partial t}=-\frac{\partial f}{\partial \phi},
$$

where $\gamma$ is the rotational viscosity for reorientation on the smectic cone. The model is based on the assumption of a spatially uniform director field that implies neglecting elasticity effects along with the coupling between the fluid flow and the director.

Such uniform theory suggests applying the viscositylimited dynamics to the switching process in SSFLC cells. The simplest case where the anchoring energy is disregarded was originally considered in [5,6] (see, e.g., [36] for a review). An attempt to fit experimental data using this simplified model was recently made in [16].

Subsequently, various modifications of the model such as including an effective elasticlike term [10] and the anchoring energy $[11,30,33]$ have been employed to interpret experimental results. The approach to grey levels in FLC displays based on the uniform theory is presented in [15].

In our case there are two characteristic time scales,

$$
t_{W}=\frac{\gamma d}{W}, \quad t_{E}=\frac{\gamma d}{P_{\mathrm{S}} V_{m}},
$$

and the governing equation (19) can be written in the following explicit form:

$$
\begin{gathered}
\frac{\partial \phi}{\partial \tau}=-\left[r(\tau)+\cos \phi+w_{p}\right] \sin \phi, \\
\tau=t / t_{W}, \quad r(\tau)=r_{e} v(\tau),
\end{gathered}
$$

where $r_{e}=t_{W} / t_{E}=P_{S} V_{m} / W$ is the driving voltage parameter. Note that rescaling of time renormalizes the period of the wave-form function $v(\tau): T \rightarrow T_{w}=T / t_{W}$.

Equation (21) presents the simplest model that can be used to study the effects of asymmetry induced by the polar anchoring energy. Our main interest is with the characteristics of the hysteresis loop depicted in Fig. 3.

Referring to Fig. 3, the location of the peaks of the polarization reversal current is determined by two switching voltage thresholds, $V_{+}=V_{m} v_{+}$and $V_{-}=-V_{m} v_{-}$, where $v_{ \pm}$is the 

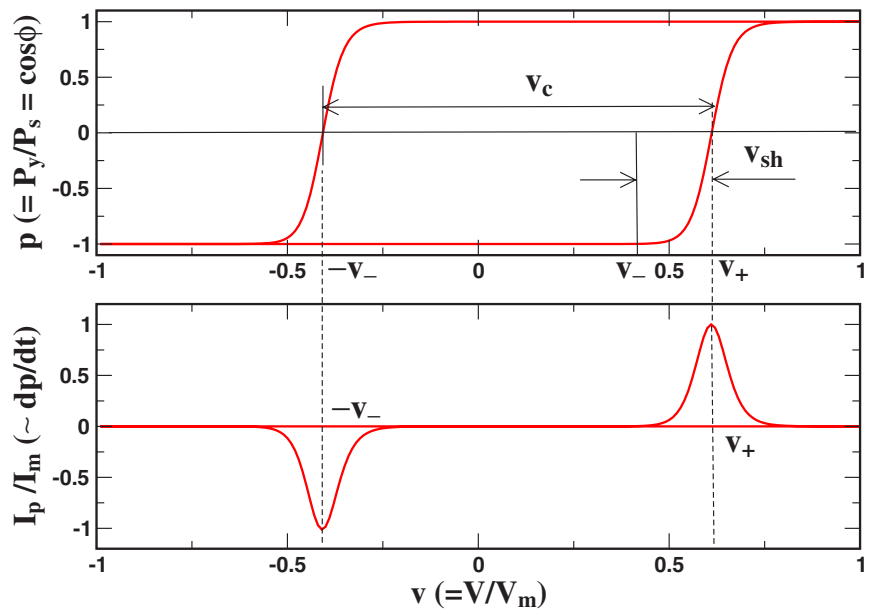

FIG. 3. (Color online) Hysteresis loop.

dimensionless switching voltage parameter, characterizing the hysteresis loop for the normalized polarization (polarization parameter), $p=P_{y} / P_{\mathrm{S}}=\cos \phi$. Equivalently, the width and the shift of the loop can be conveniently described by the dimensionless (normalized) voltage parameters

$$
v_{c}=v_{+}+v_{-}, \quad v_{\mathrm{sh}}=v_{+}-v_{-},
$$

where $v_{c}$ is the voltage coercitivity and $v_{\mathrm{sh}}$ is the voltage shift.

If $w_{p}=0$, the dynamic equation (21) is invariant under the symmetry transformation: $r \rightarrow-r\left(\tau \rightarrow \tau+T_{w} / 2\right)$ and $\phi \rightarrow \pi$ $-\phi(p \rightarrow-p)$. Under these circumstances, the hysteresis loop is symmetric and the voltage shift vanishes, $v_{\mathrm{sh}}=0$. This is no longer the case when $w_{p} \neq 0$ and the symmetry is broken. For the cell driven by the sine-wave voltage at $w_{p}=0.2$, the curves representing temporal evolution of the polarization parameter, $p$, and its time derivative are shown in Fig. 4.
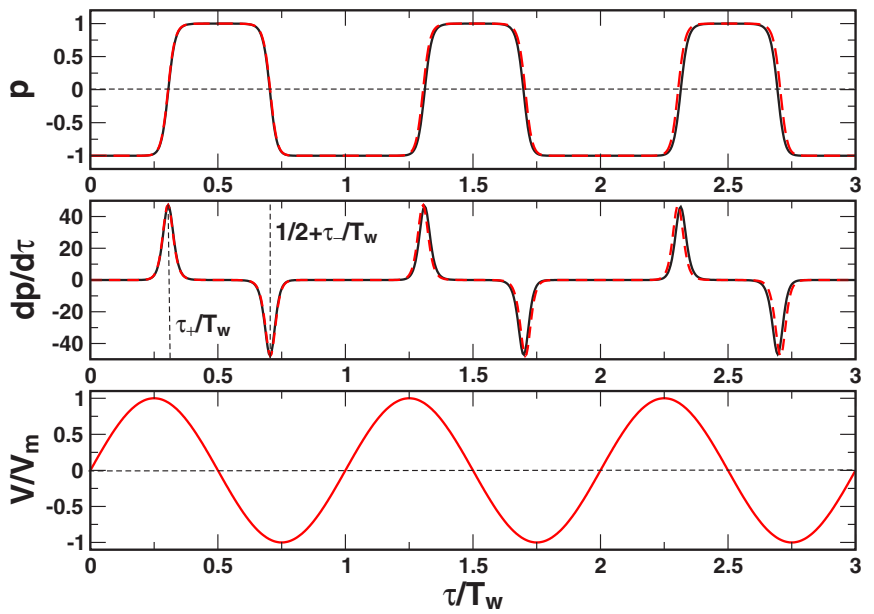

FIG. 4. (Color online) Normalized polarization and polarization reversal current evolving in time under sine-wave driving voltage at $w_{p}=0.2, T_{w}=0.8$, and $r_{e}=50$. Solid lines represent the results obtained by solving Eq. (21) numerically. Analytical results (dashed lines) are computed using the piecewise linear approximation (36).

\section{B. Switching voltages vs frequency: Simplified model}

It is instructive to examine first predictions of the extremely simple model in which the terms describing the anchoring energy (8) are disregarded and dynamics of the azimuthal angle $\phi$ is governed by the simplified equation of motion

$$
\gamma \frac{\partial \phi}{\partial t}=-P_{S} E_{0} v(t) \sin \phi
$$

Our task is to study the switching voltages as functions of the driving voltage frequency, $f=1 / T$. We shall perform analysis of the model (24) in the form suitable for subsequent generalization and clarify the assumptions underlying the results previously reported in Refs. [37,38].

We start with introducing an auxiliary angular variable $u$ linked to the azimuthal angle through the relations

$$
2 u=\ln \left(\frac{1+\cos \phi}{1-\cos \phi}\right), \quad \cos \phi \equiv p=\tanh (u)
$$

and governed by the equation $\partial_{\tau} u=v(\tau)$, where $\tau=t / t_{E}$. The latter immediately gives the normal component of the polarization vector, $P_{y}$, evolving in time as follows:

$$
\begin{gathered}
P_{y}(\tau)=P_{\mathrm{S}} \cos \phi(\tau)=P_{\mathrm{S}} \tanh [u(\tau)], \\
u(\tau)=-u_{0}+w(\tau)=-u_{0}+\int_{0}^{\tau} v\left(\tau^{\prime}\right) d \tau^{\prime},
\end{gathered}
$$

where the initial condition $\cos \phi(0)=-\tanh u_{0}$ with $u_{0}>0$ means that the cell is initially in the down state.

In order for the polarization (26) to be periodic in time the condition $w\left(T_{e}\right)=0$, where $T_{e}=T / t_{E}$, must be fulfilled. It is not difficult to see that, for bipolar switching with the waveform functions (4)-(6), this periodicity condition is satisfied.

From Eq. (27) the angular variable $u$ monotonically increases from $-u_{0}$ to $u_{+}=-u_{0}+w\left(T_{e} / 2\right)$ over the first halfperiod. Complete switching occurs when $\cos \phi_{0}=-\tanh u_{0}$ $\approx-1$ and $\cos \phi_{+}=\tanh u_{+} \approx 1$. During the switching process the polarization increases (decreases) passing zero at the instant of time $\tau_{+}\left(\tau_{-}\right)$. So, the switching voltage parameters, $v_{+}$and $v_{-}$, can be defined as follows:

$$
v_{ \pm}= \pm v\left(\tau_{ \pm}\right), \quad u\left(\tau_{ \pm}\right)=0 .
$$

For sine-wave and triangular voltages, the switching voltages (28) are given by

$$
\begin{aligned}
& v_{ \pm}^{(\text {sine })}=\sqrt{u_{0} \omega_{e}\left(2-u_{0} \omega_{e}\right)}, \quad u_{0} \omega_{e} \leqslant 1, \quad \omega_{e}=2 \pi / T_{e}, \\
& v_{ \pm}^{\text {(triang) }}= \begin{cases}\sqrt{8 u_{0} / T_{e}}, & 0 \leqslant u_{0} / T_{e} \leqslant 1 / 8, \\
\sqrt{2-8 u_{0} / T_{e}}, & 1 / 8 \leqslant u_{0} / T_{e} \leqslant 1 / 4 .\end{cases}
\end{aligned}
$$

It would appear natural that the polarization in the up and down states is of the same magnitude but differs in sign. In this case, the parameter $u_{0}$, 


$$
2 u_{0}=w\left(T_{e} / 2\right)
$$

is fixed by the symmetry condition $\cos \phi_{+}=-\cos \phi_{0}\left(u_{+}=u_{0}\right)$. Since $w_{\text {sine }}\left(T_{e} / 2\right)=2 / \omega_{e}$ and $w_{\text {triang }}\left(T_{e} / 2\right)=T_{e} / 4$, we are led to the conclusion that the switching voltages are frequency independent and $v_{ \pm}^{\text {(sine) }}=v_{ \pm}^{\text {(triang) }}=1$.

As a way around this difficulty, we can apply a cutoff procedure where the equilibrium states are regarded as "saturated" states characterized by the cutoff parameter $u_{s}$, $\tanh u_{s} \approx 1$. The result is that the relation between the angular and polarization parameters takes the modified form

$$
\cos \phi= \begin{cases}\tanh u, & |u| \leqslant u_{s}, \\ \operatorname{sign}(u) \tanh u_{s} \equiv \pm \cos \phi_{s}, & |u| \geqslant u_{s}\end{cases}
$$

and the parameter $u_{0}$ is now given by

$$
2 u_{0}= \begin{cases}w\left(T_{e} / 2\right), & T_{e} \leqslant T_{s}, \\ 2 u_{s}=w\left(T_{s} / 2\right), & T_{e} \geqslant T_{s} .\end{cases}
$$

From Eq. (33) it can be seen that the cutoff parameter $u_{s}$ determines the boundary frequency, $f_{s}=1 / T_{s}$, separating the regions of low and high frequencies. In the low frequency regime with $f_{e}=1 / T_{e}<f_{s}$, the switching voltage parameters are given by

$$
v_{ \pm}^{(\text {sine })}=\sqrt{\left(2-\omega_{e} / \omega_{s}\right) \omega_{e} / \omega_{s}}, \quad v_{ \pm}^{\text {(triang) }}=\sqrt{f_{e} / f_{s}},
$$

where $\omega_{s}=2 \pi f_{s}$.

Similar to the switching voltages, the switching time parameters, $\tau_{+} / T_{e}$ and $\tau_{-} / T_{e}$, are frequency independent, $\tau_{+} / T_{e}=1-\tau_{-} / T_{e}=1 / 4$, at $f_{e}>f_{s}$ for all three wave forms (4)-(6). For the square-wave driving voltage of low frequency, these parameters assume linear dependence on the frequency: $\tau_{+} / T_{e}=1-\tau_{-} / T_{e}=f_{e} /\left(4 f_{s}\right)$.

\section{SWITCHING DYNAMICS}

From the analysis presented in Sec. II B it can be inferred that the properties of switching dynamics are predominately determined by a certain class of time-periodic solutions to the governing equation. Specifically, we have used the symmetry condition $p_{+}=-p_{-}$, where $p_{+}\left(p_{-}\right)$is the polarization parameter of the up (down) state, in combination with the cutoff procedure.

Now we pass onto the model (21) and study how the anchoring energy influences the dynamics of switching. As in the preceding section, our attention will be focused on the frequency dependence of the switching voltages in the regime of complete switching.

By contrast to Eq. (24), the dynamic equation (21) is generally not exactly solvable. Additional difficulties emerge as far as analysis of the solutions describing the switching process is concerned. These solutions need to be periodic in time and will be referred to as the cycles. As we shall be seeing later in this section the very existence of cycles does not follow from Eq. (21) immediately and the periodicity conditions may require somewhat involved considerations.

In this section we present an analytical approach to study the cycles based on approximating the dynamic equation for the angular variable $u$ defined in Eq. (25). By applying this method, the case of square wave voltages can be treated analytically, whereas a relatively simple numerical analysis is needed for other wave forms.

As a first step, we shall write the equation for the angular variable $u$

$$
\frac{\partial u}{\partial \tau} \equiv \dot{u}=\tanh (u)+\left[r(\tau)+w_{p}\right], \quad p \equiv \cos \phi=\tanh (u),
$$

deduced from Eq. (21) by using Eq. (25) to make the change of variables: $\phi \rightarrow u$. Then we apply the piecewise linear approximation for the hyperbolic tangent

$$
\tanh (u) \rightarrow L(u)= \begin{cases}u, & |u|<1, \\ \operatorname{sgn}(u), & |u| \geqslant 1\end{cases}
$$

and obtain the approximate dynamic equation

$$
\dot{u}=L(u)+\left[r(\tau)+w_{p}\right]
$$

that will be the starting point for our subsequent considerations.

Equation (37) retains the symmetry of Eq. (35) discussed at the end of Sec. II A. It can be readily checked that the transformation

$$
\tau \rightarrow \tau-T_{w} / 2, \quad u \rightarrow-u, \quad w_{p} \rightarrow-w_{p}
$$

keeps both of these equations intact.

It turned out that, as far as the cycles are concerned, the numerical results computed from Eq. (35) and the predictions of the approximate model (37) are essentially the same. In our calculations the relative error was found to be well below $0.1 \%$.

Accuracy of the approximation is demonstrated in Fig. 4. It is seen that the difference between the solid and dashed lines becomes noticeable only with a rise in numerical integration error as the interval of integration increases.

As in Sec. II B, suppose that the cell is initially in the down state with $u(0)=-u_{0}$ and $u_{0}>1$. After the first halfperiod time, the external voltage drives the cell into the up state with $u\left(T_{w} / 2\right)=u_{+}$. This process might be called switching up and we consider it complete if $u_{+}>1$. Analogously, complete switching down takes place over the second halfperiod time when the cell goes from the up state with $u\left(T_{w} / 2\right)=u_{+}$to the down state with $u\left(T_{w}\right)=-u_{-}$and $u_{-}>1$.

Mathematically, this can be described in terms of the halfperiod mappings

$$
\begin{gathered}
\Psi_{+}: u_{0}=-u(0) \mapsto u_{+}=u\left(T_{w} / 2\right), \\
\Psi_{-}: u_{+}=u\left(T_{w} / 2\right) \mapsto u_{-}=-u\left(T_{w}\right),
\end{gathered}
$$

where $\Psi_{+}$and $\Psi_{-}$are determined by temporal evolution of the angular variable $u$ over the first and second half-period, respectively.

Owing to the symmetry (38), $\Psi_{-}$can be obtained from $\Psi_{+}$ by changing the sing of the polar anchoring parameter $w_{p}$. So, in the subsequent section, we concentrate on the halfperiod mapping $\Psi_{+}$. 


\section{A. Half-period mappings and cycles}

We restrict ourselves to the first half-period and study in detail how the angular parameter $u$ evolves in time. For complete switching up, the solution can be written in the following general form:

$$
u(\tau)= \begin{cases}F_{0}(\tau), & \tau<\tau_{0}, \\ F(\tau), & \tau_{0} \leqslant \tau \leqslant \tau_{1}, \\ F_{+}(\tau), & \tau_{1}<\tau \leqslant T_{w} / 2,\end{cases}
$$

where $0<\tau_{0}<\tau_{1} \leqslant T_{w} / 2$ are defined by the conditions

$$
u\left(\tau_{0}\right)=-1, \quad u\left(\tau_{1}\right)=1 .
$$

It is not difficult to solve Eq. (37) and derive the explicit formulas for the functions that enter the solution (40). The result is

$$
\begin{gathered}
F_{0}(\tau)=-u_{0}+\left(w_{p}-1\right) \tau+R_{0}(\tau) \\
F(\tau)=\exp \left(\tau-\tau_{0}\right)\left(w_{p}-1\right)-w_{p}+\exp (\tau)\left[R\left(\tau_{0}\right)-R(\tau)\right] \\
F_{+}(\tau)=1+\left(w_{p}+1\right)\left(\tau-\tau_{1}\right)+R_{0}(\tau)-R_{0}\left(\tau_{1}\right)
\end{gathered}
$$

where

$$
R_{0}(\tau)=\int_{0}^{\tau} r\left(\tau^{\prime}\right) d \tau^{\prime}, \quad R(\tau)=-\int_{0}^{\tau} \exp \left(-\tau^{\prime}\right) r\left(\tau^{\prime}\right) d \tau^{\prime} .
$$

From Eqs. (40)-(44) the half-period mapping $\Psi_{+}$from $u_{0}$ to $u_{+}$can be derived in the parametrized form

$$
\begin{gathered}
\Psi_{+}: u_{0}-1=G_{0}\left(\tau_{0}\right)=\left(w_{p}-1\right) \tau_{0}+R_{0}\left(\tau_{0}\right), \\
u_{+}-1=G_{+}\left(\tau_{1}\right)=\left(w_{p}+1\right)\left(T_{w} / 2-\tau_{1}\right)+R_{0}\left(T_{w} / 2\right)-R_{0}\left(\tau_{1}\right), \\
g_{-}\left(\tau_{0}\right)=g_{+}\left(\tau_{1}\right), \quad g_{ \pm}(\tau)=\exp (-\tau)\left(w_{p} \pm 1\right)+R(\tau),
\end{gathered}
$$

where the parameters $\tau_{0}$ and $\tau_{1}$ are defined by the two conditions (41). The first condition $u\left(\tau_{0}\right)=F_{0}\left(\tau_{0}\right)=-1$ and the relation $u\left(T_{w} / 2\right)=F_{+}\left(T_{w} / 2\right)=u_{+}$give $u_{0}$ and $u_{+}$as a function of $\tau_{0}$ and $\tau_{1}$, respectively [see Eqs. (46a) and (46b) for the corresponding expressions]. The parameters $\tau_{0}$ and $\tau_{1}$ are coupled through Eq. (46c) which is an immediate consequence of the second condition from Eq. (41): $u\left(\tau_{1}\right)=F\left(\tau_{1}\right)$ $=1$.

Changing the sign of $w_{p}$ in Eqs. (46a)-(46c) gives the formulas for the half-period mapping $\Psi_{-}$. Composition of the two mappings

$$
\Psi=\Psi_{-} \circ \Psi_{+}, \quad \Psi_{-}=\left.\Psi_{+}\right|_{w_{p} \rightarrow-w_{p}}
$$

relates the values of $u$ at the end points of the period: $u(0)$ $=-u_{0} \rightarrow u\left(T_{w}\right)=-u_{-}$.

From the periodicity condition $u_{0}=u_{-}$it follows that, for cycles, $u_{0}$ is the fixed point of $\Psi$. So, we have the fixed point equations

$$
\begin{gathered}
\Psi\left(q_{-}^{(\mathrm{st})}\right)=q_{-}^{(\mathrm{st})}, \quad q_{-}^{(\mathrm{st})} \equiv u_{-}^{(\mathrm{st})}-1=u_{0}^{(\mathrm{st})}-1, \\
\Psi_{+}\left(q_{-}^{(\mathrm{st})}\right)=q_{+}^{(\mathrm{st})} \equiv u_{+}^{(\mathrm{st})}-1
\end{gathered}
$$

characterizing the up and down states of the cycle. The fixed point then can be found by solving Eq. (48a). It provides the polarization parameter for the down state of the cycle: $p_{-}^{(\mathrm{st})}$ $=-\tanh u_{-}^{(\mathrm{st})}$, whereas Eq. (48b) gives $u_{+}^{\text {(st) }}$ and the polarization parameter of the up state: $p_{+}^{(\mathrm{st})}=\tanh u_{+}^{(\mathrm{st})}$.

Given the cycle Eqs. (35)-(45) with $u_{0}=u_{-}^{(\text {st) }}$ yield $u(\tau)$ for switching up during the first half-period, $0 \leqslant \tau \leqslant T_{w} / 2$. The reversal polarization current reaches a maximum at the instant of time $\tau_{+}$where $\ddot{p}\left(\tau_{+}\right)=0$.

For the second half-period time, $T_{w} / 2 \leqslant \tau \leqslant T_{w}$, Eqs. (35)-(45) with $u_{0}=u_{+}^{\text {(st) }}$ and $w_{p} \rightarrow-w_{p}$ give $-u\left(\tau-T_{w} / 2\right)$. The current is now peaked at $\tau=T_{w} / 2+\tau_{-}$. From the condition $\ddot{p}=0$ and Eq. (35) we can derive the equations for $\tau_{+}$and $\tau_{-}$ $\dot{u}\left(\tau_{ \pm}\right)\left[2 \dot{u}\left(\tau_{ \pm}\right) \tanh u\left(\tau_{ \pm}\right)-1\right] \approx \dot{u}\left(\tau_{ \pm}\right)\left[2 \dot{u}\left(\tau_{ \pm}\right) u\left(\tau_{ \pm}\right)-1\right]=\dot{r}\left(\tau_{ \pm}\right)$,

$$
\dot{u}\left(\tau_{ \pm}\right)=u\left(\tau_{ \pm}\right)+\left[r\left(\tau_{ \pm}\right) \pm w_{p}\right],
$$

where $0<\tau_{ \pm}<T_{w} / 2, u\left(\tau_{+}\right)=F\left(\tau_{+}\right)$, and $u\left(\tau_{-}\right)=\left.F\left(\tau_{-}\right)\right|_{w_{p} \rightarrow-w_{p}}$. Then the switching voltages are given by

$$
r_{ \pm}= \pm r\left(\tau_{ \pm}\right)= \pm r_{e} v_{ \pm}
$$

where $v_{ \pm}=v\left(\tau_{ \pm}\right)$is the switching voltage parameter.

\section{B. Square wave voltage}

Analytical results of the preceding section depend on the wave form through the functions defined in Eq. (45). The case of square wave voltages can be treated by replacing $w_{p}$ with $w_{p}+r_{e}$ and setting $R_{0}$ and $R$ equal to zero. The result for the functions that enter the half-period mappings [see Eqs. (46a) and (46b)] and the coupling equation (46c) is

$$
\begin{gathered}
\Psi_{ \pm}: G_{0}=\left( \pm w_{p}+r_{e}-1\right) \tau_{0}, \\
G_{+}=\left( \pm w_{p}+r_{e}+1\right)\left(T_{w} / 2-\tau_{1}\right), \\
\tau_{1}=\tau_{0}+\gamma_{ \pm}=\tau_{0}+\ln \beta_{ \pm}, \quad \beta_{ \pm}=\frac{r_{e} \pm w_{p}+1}{r_{e} \pm w_{p}-1} .
\end{gathered}
$$

By using Eqs. (52a)-(52c) along with the formulas (46a) and (46b) the parameters $\tau_{0}$ and $\tau_{1}$ can be eliminated to yield the half-period mappings, $\Psi_{+}$and $\Psi_{-}$, as the linear functions given by

$$
\begin{aligned}
& q_{+}=\Psi_{+}\left(q_{0}\right)=\alpha_{+}-\beta_{+} q_{0} \equiv q_{1}^{(+)}\left(1-q_{0} / q_{0}^{(+)}\right), \\
& q_{-}=\Psi_{-}\left(q_{+}\right)=\alpha_{-}-\beta_{-} q_{+} \equiv q_{1}^{(-)}\left(1-q_{+} / q_{0}^{(-)}\right),
\end{aligned}
$$

where $q_{i} \equiv u_{i}-1$ and

$$
q_{1}^{( \pm)} \equiv \alpha_{ \pm}=\left( \pm w_{p}+r_{e}+1\right)\left(T_{w} / 2-\gamma_{ \pm}\right), \quad q_{0}^{( \pm)}=\alpha_{ \pm} / \beta_{ \pm} .
$$

Now that the expressions for the half-period mappings are derived we can discuss some of the most important conse- 
quences. The regime of complete switching that occurs when the parameters $q_{0}=u_{0}-1, q_{+}=u_{+}-1$, and $q_{-}=u_{-}-1$ are all positive is the first point to address.

From Eq. (52a) the parameter $q_{0}=G_{0}\left(\tau_{0}\right)$ is positive at $\tau_{0}>0$ only if the driving voltage parameter, $r_{e}=P_{\mathrm{S}} V_{\mathrm{m}} / W$, exceeds its threshold value:

$$
r_{e}>1+\left|w_{p}\right| \text {. }
$$

Similarly, it can be seen from Eqs. (53a) and (53b) that the parameters $q_{+}$and $q_{-}$cannot be positive when $q_{1}^{( \pm)}$is negative. The latter and Eq. (54) give the inequality

$$
T_{w}>T_{\min }=2 \max \left(\gamma_{+}, \gamma_{-}\right)=2 \ln \frac{r_{e}-\left|w_{p}\right|+1}{r_{e}-\left|w_{p}\right|-1}
$$

that bounds the driving frequency $1 / T_{w}$ from above.

The complete switching conditions (55) and (56) define the thresholds for the amplitude and the period of the applied voltage, so that the switching is incomplete below these thresholds; but, in general, these conditions will not suffice for either completeness or periodicity of the switching regime. In other words, more stringent constraints are required to ensure that the composition of mappings (47) has fixed points describing the cycles.

In our case, the mapping $\Psi=\Psi_{-} \circ \Psi_{+}: q_{0} \rightarrow q_{-}$is linear and the parameter $q_{0}$ varies in the range from zero to $q_{0}^{(+)}$. The fixed point exists only if the function $q-\Psi(q)$ takes the values of opposite sign at the end points of the interval $\left[0, q_{0}^{(+)}\right]: q=0$ and $q=q_{0}^{(+)}$. Since $\Psi(0)=\left[q_{0}^{(-)}-q_{1}^{(+)}\right] q_{1}^{(-)} / q_{0}^{(-)}$ and $q_{0}^{(+)}-\Psi\left(q_{0}^{(+)}\right)=q_{0}^{(+)}-q_{1}^{(-)}$, the fixed point condition $\Psi(0)$ $\times\left[q_{0}^{(+)}-\Psi\left(q_{0}^{(+)}\right)\right]>0$ can be conveniently written as the inequality

$$
P_{+} P_{-}>0, \quad P_{ \pm}=q_{0}^{( \pm)}-q_{1}^{(\mp)},
$$

where the parameters $q_{0}^{( \pm)}$and $q_{1}^{( \pm)}$are defined in Eq. (54) and linearly depend on $T_{w}$.

It can be shown that $P_{+}$and $P_{-}$are both negative in the region where the cycles come into play. For this region of cycles, we have

$$
P_{ \pm}<0 \text { at } T_{w}>T_{c},
$$

where $T_{c}$ is the critical period given by

$$
T_{c}=\ln \frac{\left(r_{e}+1\right)^{2}-w_{p}^{2}}{\left(r_{e}-1\right)^{2}-w_{p}^{2}}+r_{e}\left(1-\left|w_{p}\right|\right)^{-1} \ln \frac{r_{e}^{2}-\left(1-\left|w_{p}\right|\right)^{2}}{r_{e}^{2}-\left(1+\left|w_{p}\right|\right)^{2}} .
$$

Thus there are no cycles in the high frequency region above the critical frequency $1 / T_{c}$.

According to estimates that will be discussed in Sec. IV, the voltage parameter $r_{e}$ is typically much larger than unity and the asymptotic expression

$$
T_{c} \approx 4\left(1-\left|w_{p}\right|\right)^{-1} r_{e}^{-1}
$$

can be used as a good approximation for the critical period (59) in the region of high voltage parameters where $r_{e}>10$. Clearly, $T_{c}$ declines as the voltage amplitude increases, whereas the critical period becomes divergent when the magnitude of the polar anchoring parameter approaches unity.
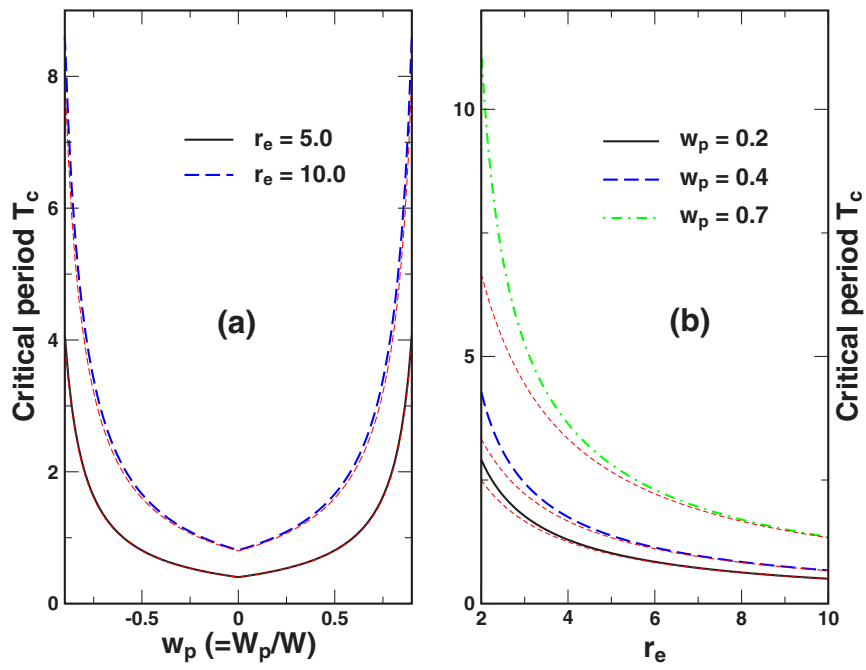

FIG. 5. (Color online) Critical period as a function of (a) polar anchoring parameter, $w_{p}=W_{p} / W$ and (b) driving voltage parameter, $r_{e}=P_{\mathrm{S}} V_{\mathrm{m}} / W$, for the square wave voltage. Thin dashed lines represent the results computed from the asymptotic formula (60).

The curves depicted in Fig. 5 illustrate these effects and accuracy of the high voltage approximation.

An important point is that, under the condition (58), the derivative of $\Psi, \Psi^{\prime}(q)=q_{1}^{(+)} q_{1}^{(-)}\left[q_{0}^{(+)} q_{0}^{(-)}\right]^{-1}$, is greater than unity. Therefore the fixed points and the cycles are unstable. Note that stability of the cycles requires both $P_{+}$and $P_{-}$to be positive.

For the half-period mappings of the form (53a) and (53b), it is straightforward to deduce the expressions for the parameters $q_{+}^{(\mathrm{st})}$ and $q_{-}^{\text {(st) }}$,

$$
q_{ \pm}^{(\mathrm{st})}=\frac{\alpha_{ \pm}-\beta_{ \pm} \alpha_{\bar{\mp}}}{1-\beta_{+} \beta_{-}}=\frac{q_{1}^{( \pm)} q_{0}^{(+)} P_{ \pm}}{q_{0}^{(+)} q_{0}^{(-)}-q_{1}^{(+)} q_{1}^{(-)}},
$$

that characterize the up and down states of the cycles. Solving Eq. (49) gives the switching time parameters $\tau_{+}$and $\tau_{-}$,

$$
\begin{gathered}
\tau_{ \pm}=q_{\mp}^{(\mathrm{st})} /\left(r_{e} \pm w_{p}-1\right)+\delta \tau_{ \pm}, \\
\delta \tau_{ \pm}=\ln \frac{r_{e} \pm w_{p}+\sqrt{\left(r_{e} \pm w_{p}\right)^{2}+2}}{2\left(r_{e} \pm w_{p}-1\right)},
\end{gathered}
$$

that determine location of the reversal current peaks in time during the first and second half-period (switching up and down). The formulas

$$
\begin{gathered}
u_{ \pm}^{(\mathrm{st})} \approx T_{w}\left(1 \mp w_{p}\right)\left[r_{e}-\left(1 \pm w_{p}\right)^{2} r_{e}^{-1}\right] / 4+2\left(1 \pm 2 w_{p}\right) r_{e}^{-2} / 3, \\
\tau_{ \pm} \approx T_{w}\left(1 \pm w_{p}\right)\left[1+\left(1 \mp w_{p}\right) r_{e}^{-1}\right] / 4 \mp 4 w_{p} r_{e}^{-3} / 3
\end{gathered}
$$

describe asymptotic behavior of $u_{ \pm}^{\text {(st) }}$ and $\tau_{ \pm}$in the region of high voltage parameters where $r_{e} \geqslant 10$.

As is expected from the symmetry (38), we have $u_{+}^{\text {(st) }}$ $=u_{-}^{(\mathrm{st})}$ and $\tau_{+}=\tau_{-}$in the limit of nonpolar anchoring with $w_{p}=0$. This is also the case where the difference between the critical period $T_{c}$ and the threshold period of complete switching $T_{\min }$ disappears. 
Interestingly, at $w_{p}=0$, the leading term of the asymptotic expansion (64) [the first term in the square brackets on the right-hand side of Eq. (64)] can be derived from the symmetry condition (31) discussed in Sec. II B. More generally, Eqs. (64) and (65) indicate that, quite similar to the high frequency regime considered in Sec. II B, the parameters $u_{ \pm}^{\text {(st) }} / T_{w}$ and $\tau_{ \pm} / T_{w}$ are almost frequency independent having only a weak linear dependence on the frequency $1 / T_{w}$.

The effects of the polar anchoring induced asymmetry depend on the polarity determined by the sign of $w_{p}$. At the critical point with $T_{w}=T_{c}$ and $w_{p}>0\left[w_{p}<0\right]$, we have $P_{+}\left(T_{c}\right)=0\left[P_{-}\left(T_{c}\right)=0\right]$ and the parameter $q_{+}^{(\mathrm{st})}\left[q_{-}^{(\mathrm{st})}\right]$ vanishes.

In the case where $w_{p}>0$ and the down state is metastable in the absence of applied voltage, for the cycles, the polarization parameter of the down state $p_{-}^{\text {(st) }}$ appears to be higher than that of the up state $p_{+}^{\text {(st) }}, p_{-}^{\text {(st) }}>p_{+}^{\text {(st) }}$. An important consequence of this is that the switching time $\tau_{+}$is longer than $\tau_{-}, \tau_{+}>\tau_{-}$and $\left(\tau_{+}-\tau_{-}\right) / T_{w} \approx w_{p} / 2$. So, at $w_{p}>0$, the model predicts that, in cycles driven by the square wave voltage, switching down is faster than switching up. Note that the results for the reversed sign of $w_{p}$ can be obtained by interchanging the up and down states.

\section{Sine-wave voltage: Numerical analysis}

Now we extend the analysis of the previous section to the case of sine-wave voltage. The triangular driving voltage can be treated in just the same way but this involves rather cumbersome expressions.

As a first step, we perform integrals in Eq. (45) for the wave-form function (4) and deduce the expressions for the functions

$$
\begin{gathered}
R(\xi)=r_{e} \exp \left(-\xi / \omega_{w}\right)\left[\omega_{w} \cos \xi+\sin \xi\right] /\left(1+\omega_{w}^{2}\right), \\
R_{0}(\xi)=r_{e}[1-\cos \xi] / \omega_{w}, \quad \xi=\omega_{w} \tau,
\end{gathered}
$$

where $\omega_{w}=2 \pi / T_{w}=\omega t_{W}$ is the dimensionless frequency parameter. Substituting Eqs. (66) and (67) into Eqs. (46a)-(46c) gives the half-period mapping $\Psi_{+}$in the following parameterized form:

$$
\begin{aligned}
\Psi_{+}: & q_{0} \equiv \omega_{w}\left(u_{0}-1\right)=\left(w_{p}-1\right) \xi_{0}+r_{e}\left[1-\cos \xi_{0}\right] \equiv G_{0}^{(\text {sine })} \\
& \times\left(\xi_{0}\right),
\end{aligned}
$$

where the parameters $\xi_{0}=\omega_{w} \tau_{0}$ and $\xi_{1}=\omega_{w} \tau_{1}$ are ranged between zero and $\pi$. (Note that the parameters $q_{i}$ differ from those defined in Sec. III B by the factor $\omega_{w}$.)

As opposed to the case of square wave voltage, the parameters $\xi_{0}$ and $\xi_{1}$ cannot be eliminated. We begin with locating the values of these parameters that represent the regime of complete switching where $q_{0}$ and $q_{+}$are nonnegative, $q_{0,+} \geqslant 0$.
Our first remark concerns behavior of the functions, $G_{0,+}$ and $g_{ \pm}$, that enter the mapping $\Psi_{+}$. The time derivatives of these functions

$$
\begin{gathered}
\frac{\partial G_{0,+}(\tau)}{\partial \tau}=-1 \pm\left[w_{p}+r(\tau)\right], \\
\frac{\partial g_{ \pm}(\tau)}{\partial \tau}=-\exp (-\tau)\left[w_{p} \pm 1+r(\tau)\right]
\end{gathered}
$$

show that over the first half-period time $G_{+}$and $g_{+}$are monotonically decreasing functions of $\tau$.

By contrast, the functions $G_{0}$ and $-g_{-}$exhibit nonmonotonic behavior. As $\tau$ increases, they decay reaching the minimum located at $\tau_{\min }$ and then grow up to the maximum at $\tau=\tau_{\max }$ decreasing at $\tau>\tau_{\max }$. The points $\tau_{\min \text { max }}$ are determined by the equation $\dot{G}_{0}(\tau)=0$ [or, equivalently, $v(\tau)=(1$ $\left.\left.-w_{p}\right) / r_{e}\right]$ and $G_{0}\left(\tau_{\min }\right)<0$.

For the sine-wave voltage, $\xi_{\min }=\arcsin \left[\left(1-w_{p}\right) / r_{e}\right]$ and $\xi_{\max }=\pi-\xi_{\min }$. The function $G_{0}^{(\text {sine) }}$ monotonically increases from $G_{0}^{(\text {sine })}\left(\xi_{\min }\right)<0$ to $G_{0}^{(\text {sine })}\left(\xi_{\max }\right)$ and it passes through zero, $G_{0}^{(\text {sine) }}\left(\xi_{0}^{(0)}\right)=0$, before the wave-form function reaches a maximum only if $G_{0}^{\text {(sine) }}(\pi / 2)>0$. The latter provides the complete switching condition for the driving voltage parameter

$$
r_{e}>\left(1+\left|w_{p}\right|\right) \pi / 2 .
$$

Interestingly, replacing $r_{e}$ with $\pi r_{e} / 2$ in Eq. (71) recovers the result for the square wave voltage given by Eq. (55).

The largest value of $\xi_{0}, \xi_{0}=\xi_{0}^{(+)}$, can be found by solving the coupling equation (68c) at $\xi_{1}=\pi$. Owing to the inequality $\xi_{0}^{(+)}>\xi_{0}^{(0)}$, the complete switching condition for the frequency is given by

$$
g_{-}\left(\xi_{0}^{(0)}\right)>g_{+}(\pi) .
$$

It can be checked that Eq. (56) with $r_{e}$ replaced by $2 r_{e} / \pi$ provides a good estimate for $T_{\min }$ defined by the condition (72).

At this stage we have found that the end points of the interval for $\xi_{0}: \xi_{0}^{(0)} \leqslant \xi_{0} \leqslant \xi_{0}^{(+)}$, can be evaluated as solutions of the equations

$$
g_{-}\left(\xi_{0}^{(+)}\right)=g_{+}(\pi), \quad G_{0}^{(\text {sine })}\left(\xi_{0}^{(0)}\right)=0 .
$$

According to the coupling equation (68c), when $\xi_{0}$ increases from $\xi_{0}^{(0)}$ to $\xi_{0}^{(+)}$, the parameter $\xi_{1}$ changes from $\xi_{1}^{(+)}$to $\pi$. The equation for $\xi_{1}^{(+)}$is

$$
g_{+}\left(\xi_{1}^{(+)}\right)=g_{-}\left(\xi_{0}^{(0)}\right) .
$$

For the parameters $q_{0}$ and $q_{+}$, it can be inferred from the above results that $q_{+}$monotonically decays from $q_{1}^{(+)}$to zero as $q_{0}$ varies from zero to $q_{0}^{(+)}$. The values of $q_{0}^{(+)}$and $q_{1}^{(+)}$can be computed from the relations

$$
q_{0}^{(+)}=G_{0}^{(\text {sine) }}\left(\xi_{0}^{(+)}\right), \quad q_{1}^{(+)}=G_{+}^{(\text {sine })}\left(\xi_{1}^{(+)}\right),
$$

where $\xi_{0}^{(+)}$and $\xi_{1}^{(+)}$are given in Eqs. (73) and (74), respectively. 
TABLE I. Parameters of the FLC mixtures measured in the Sm-C ${ }^{*}$ phase at $23{ }^{\circ} \mathrm{C}$.

\begin{tabular}{|c|c|c|c|c|c|c|}
\hline Mixture name & Pha & e sequence & & $\begin{array}{c}P_{\mathrm{S}} \\
\left(\mathrm{nC} / \mathrm{cm}^{2}\right)\end{array}$ & $\begin{array}{c}\text { Tilt angle } \\
\theta \\
\text { (deg) }\end{array}$ & $\begin{array}{c}\text { Viscosity } \\
\gamma \\
(\mathrm{Pa} \mathrm{s})\end{array}$ \\
\hline FLC-497 & $\mathrm{Cr} \stackrel{4^{\circ} \mathrm{C}}{\longrightarrow} \mathrm{Sm}-\mathrm{C}^{*}$ & $\stackrel{57{ }^{\circ} \mathrm{C}}{\longrightarrow} \mathrm{Sm}-\mathrm{A}^{*}$ & $\stackrel{76^{\circ} \mathrm{C}}{\longrightarrow}$ Iso & 95 & 27 & 0.11 \\
\hline FLC-510 & $\mathrm{Cr} \stackrel{2{ }^{\circ} \mathrm{C}}{\longrightarrow} \mathrm{Sm}-\mathrm{C}^{*}$ & $\stackrel{71^{\circ} \mathrm{C}}{\longrightarrow} \mathrm{Sm}-\mathrm{A}^{*}$ & $\stackrel{99{ }^{\circ} \mathrm{C}}{\longrightarrow}$ Iso & 98 & 31 & 0.18 \\
\hline
\end{tabular}

So, we arrive at the conclusion that the parametrized mapping (68) behaves just like the half-period mapping for the square wave voltage (53a). In addition, numerical calculations show that using the formulas (53) as a linear approximation for the parametrized mappings $\Psi_{+}$and $\Psi_{-}$does not introduce any noticeable errors. [The relative error is typically below $10^{-4}$.]

It immediately follows that the fixed point condition (57) and Eq. (61) remain applicable for the sine-wave voltages. However, the switching time parameters $\xi_{+}$and $\xi_{-}$can only be found numerically by solving Eq. (49) where the relations (66) and (68a) are used to define the function (43) and to evaluate the values of $\xi_{0}$ for $q_{ \pm}^{\text {(st) }}$, respectively.

For the most part, qualitative predictions of the model for the sine-wave voltage remain unaltered as compared to the case of the square wave voltage. The cycles are found to be unstable and the condition (58) determines the cycle region. Despite that the critical period is longer than $T_{c}$ given by Eq. (59), its dependence on the voltage and polar anchoring parameters is qualitatively the same.

Similar to Eq. (64), the $T$-dependence of the parameters $u_{ \pm}^{\text {(st) }}$ turned out to be approximately linear, whereas the switching time parameters $\xi_{ \pm}$show a weak dependence on the frequency. The effects of polarity are analogous to those discussed at the end of Sec. III B.

Thus for all the wave forms under consideration, the fixed points of the mapping (47) appear to be repelling. This indicates instability of the cycles as the characteristic feature of the model (35). It means that initially small deviations from a cycle will grow in time.

In Ref. [39], in order to clarify the mechanism of such instability, we have extended our analysis to the generalized dynamic model with the double-well potential that can be regarded as a continuous deformation of the original model (35) deduced for the anchoring potential (8) taken in the Rapini-Papoular form. The results of Ref. [39] suggest that the shape of the effective potential characterizing a uniform model may have a profound effect on the properties of the periodic switching regimes. In particular, it was found that the branch of stable cycles emerges even at small deformations of the potential. In Sec. V we shall discuss some of the results at greater length.

\section{EXPERIMENT}

In this section we present the experimental results on frequency dependence of the threshold voltages measured in asymmetric SSFLC cells. It is found that the asymmetry in- duced shift of the hysteresis loop increases with the driving frequency. We apply the theoretical results of Sec. III to model the process of switching within the cells and to estimate the polar anchoring parameter from the experimental data.

\section{A. Sample preparation}

In our experiments we used asymmetric FLC cells where the FLC layer is sandwiched between two dissimilar substrates. One of the substrates was covered with a photoaligning substance, the azobenzene sulfric dye SD-1, whereas the other one was simply washed in $N, N$-dimethylformamide (DMF) and covered with calibrated spacers. By contrast, the substrates with photoaligned films identical in anchoring properties were assembled to form symmetric FLC cells.

Following the procedure described in Ref. [40], SD-1 was synthesized from corresponding benzidinedisulfonic acid using azo coupling. The solution was spin-coated onto glass substrates with indium-tin-oxide (ITO) electrodes at $800 \mathrm{rpm}$ for $5 \mathrm{~s}$ and, subsequently, at $3000 \mathrm{rpm}$ for $30 \mathrm{~s}$. The solvent was evaporated on a hot plate at $140{ }^{\circ} \mathrm{C}$ for $10 \mathrm{~min}$.

The surface of the coated film was illuminated with linearly polarized UV light using a super-high-pressure $\mathrm{Hg}$ lamp through an interference filter at the wavelength $365 \mathrm{~nm}$ and a polarizing filter. The intensity of light irradiated normally on the film surface during 30 min was $6 \mathrm{~mW} / \mathrm{cm}^{2}$.

We used two different pitch-compensated liquid crystal mixtures: FLC-497 and FLC-510 (from P. N. Lebedev Physical Institute of Russian Academy of Sciences) as materials for the FLC layer. The mixtures were injected into the cells in the isotropic phase by capillary action at $T=85$ and $100{ }^{\circ} \mathrm{C}$ for FLC-497 and FLC-510, respectively. In these mixtures, the FLC helix is unwound due to compensation by two chiral dopants with opposite sense of chirality (opposite sign of handedness) but the same sign of the spontaneous polarization [41]. The parameters of the mixtures are listed in Table I.

\section{B. Experimental results and modeling}

Measurements of the hysteresis loops were performed using the standard electro-optical setup composed of a $\mathrm{He}-\mathrm{Ne}$ laser, a Hewlett Packard Infinum digital oscilloscope, and a generator of triangular pulses. We also used a rotating table for adjusting the angular position of the FLC cell placed between crossed polarizers. The voltage amplitude of the generator, $V_{\mathrm{m}}$, used in all experiments was $10 \mathrm{~V}$ and the 

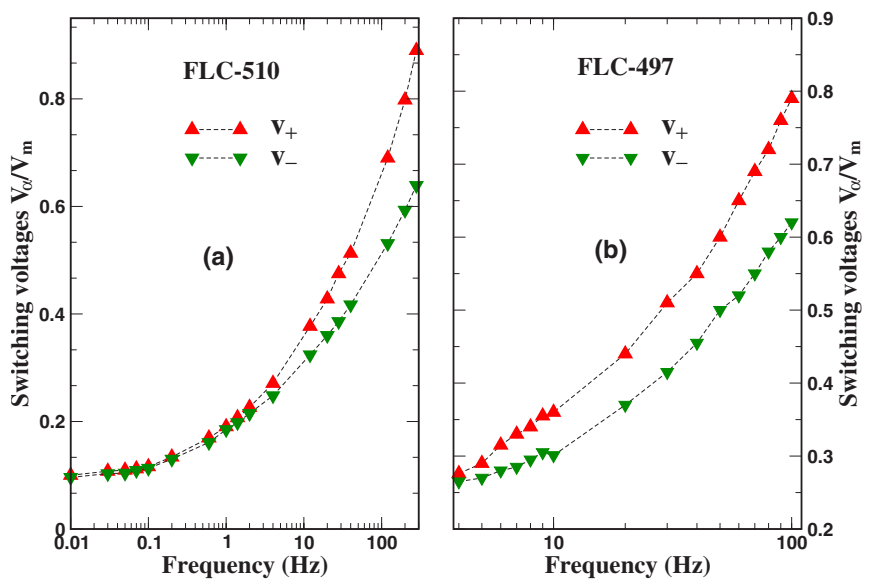

FIG. 6. (Color online) Experimental curves for switching voltage parameters, $v_{ \pm}= \pm V_{ \pm} / V_{\mathrm{m}}$, measured as a function of frequency in the cells filled with (a) FLC-510 and (b) FLC-497.

driving voltage frequency can be varied in the range from $10^{-3}$ to $10^{3} \mathrm{~Hz}$.

Figure 3 schematically represents the form of a typical hysteresis loop measured in our experiments. The threshold voltages, $V_{ \pm}$, and the corresponding values of the switching voltage parameters, $v_{ \pm}= \pm V_{ \pm} / V_{\mathrm{m}}$, then can be extracted from the experimental data for the electro-optic hysteresis loops. For asymmetric cells filled with FLC-510 and FLC-497, the results shown in Fig. 6 clearly indicate the difference between the voltage parameters for switching up and down, $v_{+}$ and $v_{-}$. Therefore the hysteresis loops are shifted. By contrast, for symmetric cells, the shift, $v_{+}-v_{-}$, is found to be vanishing within the limits of experimental error.

The curves presented in Fig. 6 were measured in the cells where the thickness of the FLC layer, $d$, and of the aligning film, $d_{A}$, was $5 \mu \mathrm{m}$ and $12 \mathrm{~nm}$, respectively. In our previous papers [42-44], we studied anchoring properties of the azodye films and our experimental technique can be applied to measure the strength of anchoring $W$ in FLC cells. It was estimated to be about $2 \times 10^{-4}$ and $1.2 \times 10^{-4} \mathrm{~J} \mathrm{~m}^{-2}$ in the cells filled with FLC-510 and FLC-497, respectively.

The characteristic times $t_{E}$ and $t_{W}$ given in Eq. (20) can now be estimated as follows: $t_{E} \approx 0.9 \times 10^{-4} \mathrm{~s}$ and $t_{W} \approx 4.5$ $\times 10^{-3} \mathrm{~s}$ for the FLC-510 cell; $t_{E} \approx 0.58 \times 10^{-4} \mathrm{~s}$ and $t_{W}$ $\approx 4.58 \times 10^{-3} \mathrm{~s}$ for the FLC-497 cell. The corresponding values of the driving voltage parameter are $r_{e} \approx 50$ and $\approx 79$.

The above estimates define the parameters that enter the dynamic equation (21) of the model discussed in Sec. II A. In this model the director field (1) is assumed to be uniform across the cell. Such an assumption can be a reasonable approximation in the high field regime of switching. For square wave voltages, this is the case in which the characteristic length $\xi_{E}=\left(K / P_{S} E_{0}\right)^{1 / 2}$, where $K$ is the effective elastic constant, is shorter than the cell thickness, $\xi_{E}<d$. From the estimate $\xi_{E} \approx 0.07 \mu \mathrm{m} \ll d=5.0 \mu \mathrm{m}$ obtained using a typical value of the elastic constant, $K \approx 10^{-11} \mathrm{~J} \mathrm{~m}^{-1}$, it might be concluded that the uniform model can be safely used to interpret the experimental results. For triangular and sinusoidal wave forms, the above estimate means that the inequality $\xi_{E} \ll d$ is satisfied during most of the driving voltage period.
We can also estimate the anchoring extrapolation length $\xi_{W}=K / W \approx 0.05-0.08 \mu \mathrm{m}$, so that the characteristic lengths $\xi_{E}$ and $\xi_{W}$ are of the same order. Therefore the anchoring conditions at the boundary surfaces described by the anchoring energy could play an important part in the switching dynamics.

In Sec. II A, we found the expression for the electric field inside the FLC layer (15) which differ from the external electric field due to the presence of the insulating aligning film. This effect crucially depends on the value of the dimensionless parameter $\eta$ defined in Eq. (13) that, in our case, can be estimated by assuming that $d_{2}=0, d_{1}=d_{A}=12 \mathrm{~nm}$, and $\varepsilon_{1}=\varepsilon_{A} \approx 7.5$ is the dielectric constant of the SD-1 layer. For both FLC-510 and FLC-497 cells, we obtain small values of $\eta$ that are below $5 \times 10^{-4}$. As a result, even a rough estimate for the depolarizing voltage $V_{\mathrm{dep}} \approx P_{\mathrm{S}} d_{A} /\left(\varepsilon_{A} \varepsilon_{0}\right) \approx 0.16 \mathrm{~V}$ derived from Eq. (15) yields the voltages an order of magnitude lower than typical values of the voltage shift measured in our experiments. In addition, a more accurate analysis of the contributions to the effective electrostatic free energy (16) clearly shows that the voltage drop across the aligning layer cannot be responsible for asymmetry of the switching voltage thresholds, $V_{+} \neq V_{-}$.

The method developed in Sec. III can now be applied to model switching dynamics of the FLC cells using the experimental results. To this end we calculated the parameters $u_{ \pm}$ characterizing the polarization of the up and down states from the data on frequency dependence of the switching voltage parameters $v_{ \pm}$. This procedure involves solving Eq. (49) and using the half-period mapping (46) for the triangular wave-form function (5). The mapping $\Psi_{+}\left(\Psi_{-}\right)$is used to compute the parameters $u_{ \pm}$for switching up (down) from the data on the switching voltage $v_{+}\left(v_{-}\right)$.

In general, the computed values of $u_{ \pm}$for switching up deviate from the corresponding results for switching down. We used the polar anchoring parameter $w_{p}$ as a fitting parameter to minimize the difference and to obtain the results nearest to the cycle. For the FLC-510 cell, the value of $w_{p}$ is found to be about -0.65 and we present the results for the parameters $\left(u_{ \pm}-1\right) f_{w}$ in Fig. 7. Referring to Fig. 7, there are two frequency regions where, similar to cycles, the frequency dependence of $\left(u_{ \pm}-1\right) f_{w}$ is nearly linear. Using linear approximation for the parameters $\left(u_{ \pm}-1\right) f_{w}$ we estimated the boundary frequency separating the low and high frequency regions at about $16 \mathrm{~Hz}$ and calculated the curves for the switching voltage parameters shown in Fig. 8(a) as solid lines.

The switching voltage vs frequency curves computed along the same lines for the FLC-497 cell are given in Fig. 8(b). In this case, the polar anchoring parameter is found to be about -0.78 . From Fig. 9(a) it can be seen that the linear approximation in the high frequency region reproduces the experimental data for the voltage coercitivity remarkably well. The scatter in the data and its scale are more evident in the results for the voltage shift shown in Fig. 9(b).

Despite good agreement between the theoretical and the experimental results, it is essential that the linearly fitted data, strictly speaking, do not represent the cycle and the modeling was only performed over the first period. Since the 

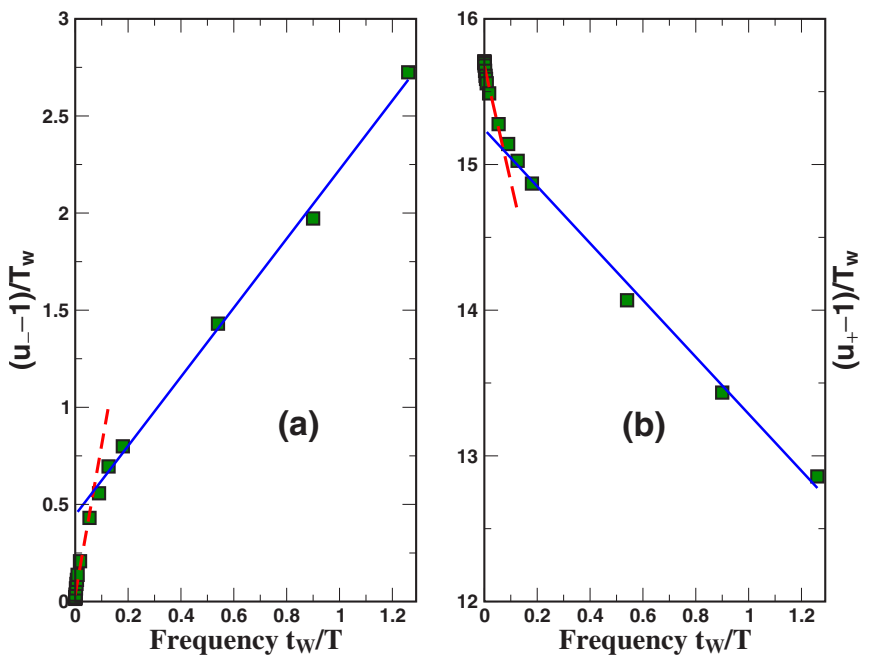

FIG. 7. (Color online) Frequency dependence of parameters (a) $\left(u_{-}-1\right) / T_{w}$ and (b) $\left(u_{+}-1\right) / T_{w}$ computed from the experimental data for the FLC-510 (squares) cell using the model (37) with $r_{e}$ $=50$ and $w_{p}=-0.65$. [The parameter $u_{ \pm}$determines the polarization of the up (down) state $P_{ \pm}= \pm P_{\mathrm{S}} \tanh u_{ \pm}$.] Piecewise linear approximation of the results defines the ranges of high (solid lines) and low (dashed lines) frequencies with the boundary frequency about $0.07 / t_{W} \approx 16 \mathrm{~Hz}$.

cycles are unstable, evolution in time will have the deterioration effect on the agreement between modeling and measurements.

\section{DISCUSSION AND CONCLUSIONS}

In this paper we have studied how the dynamical characteristics of switching in SSFLC cells are affected by asymmetry of dissimilar substrates in anchoring properties. The asymmetry effects are found to be dominated by the polar contribution to the anchoring energy and manifest them-
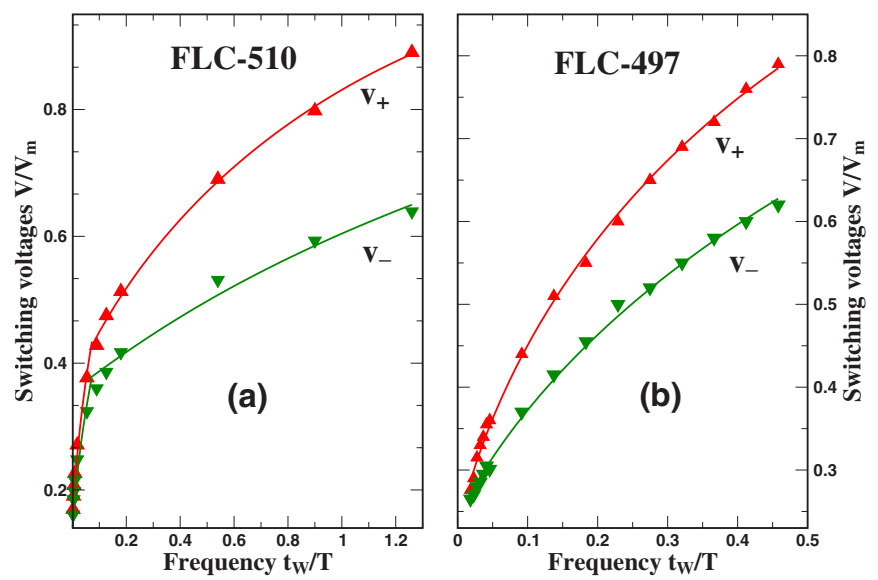

FIG. 8. (Color online) Switching voltage parameter computed as a function of frequency (solid lines) using linearly approximated frequency dependence of $\left(u_{ \pm}-1\right) f_{w}$ for (a) the FLC-510 cell and (b) the FLC-497 cell. The experimental data are shown by solid triangles. selves in the frequency dependent shift of the hysteresis loop.

Our theoretical considerations were primarily concerned with the predictions of the uniform theory on the frequency dependence of the switching voltage thresholds (the switching voltage parameters). It was assumed that the steady state regime of switching is determined by the cycles which are time-periodic solutions to the dynamic equation. So, we have developed the method to analyze the properties of cycles depending on the driving frequency. In this method the dynamic equation for the angular variable (25) is approximated to yield the half-period mappings in the parametrized form. The cycles are then studied in terms of the fixed points of the composition of two half-period mappings.

By using this method we have performed analyses for the cases of square wave, sine-wave, and triangular voltages. It was found that the cycles are unstable and can only be formed when the driving frequency is lower than its critical value, $f<f_{c}=1 / T_{c}$. The critical frequency $f_{c}$ declines with the polar anchoring parameter $w_{p}$ suppressing the cycles at $\left|w_{p}\right|=1$.

The polar anchoring breaks the mirror symmetry relating the processes of switching up and down leading to the difference in the magnitude of the corresponding switching time and switching voltage parameters. So, we have $\tau_{+} \neq \tau_{-}$ and $v_{+} \neq v_{-}$at nonvanishing polar anchoring parameter, $w_{p}$ $\neq 0$. Therefore the shift of the hysteresis loop, $v_{+}-v_{-}$, results from the symmetry breaking effect caused by the polar contribution to the anchoring potential (8) characterized by the polar anchoring parameter (9).

Our calculations indicate that, for cycles, dependence of

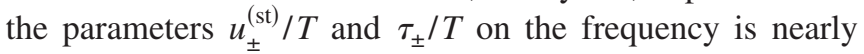
linear. Modeling of the switching dynamics using the experimental data revealed similar behavior provided the frequency is not too low. Since the conductivity of the FLC layer has been neglected in the electrostatic model discussed in Sec. II A, we cannot expect the model to give accurate results at low frequencies.

The expression for the effective electrostatic potential (16) shows that there are no symmetry breaking contributions due to the voltage drop across the insulating aligning films and the dielectric anisotropy of the FLC layer. So, they cannot be responsible for the asymmetry effects under consideration. Thus we may conclude that the anchoring energy is the determining factor in this problem. Of interest is the fact that a similar conclusion underlines the method proposed in [45] to control the process of switching.

One of the most important above results is that the fixed points describing the periodic regimes of response of periodically driven FLC cells are repelling. Therefore the model (37) predicts instability of the cycles.

Such instability can be assumed to be a characteristic of the model with the potential taken in the standard RapiniPapoular form [25]. Interestingly, our analytical approach can be used to demonstrate that the shape of the anchoring potential may affect the switching dynamics significantly [39].

To this end, we have studied the generalized model with the governing equation 

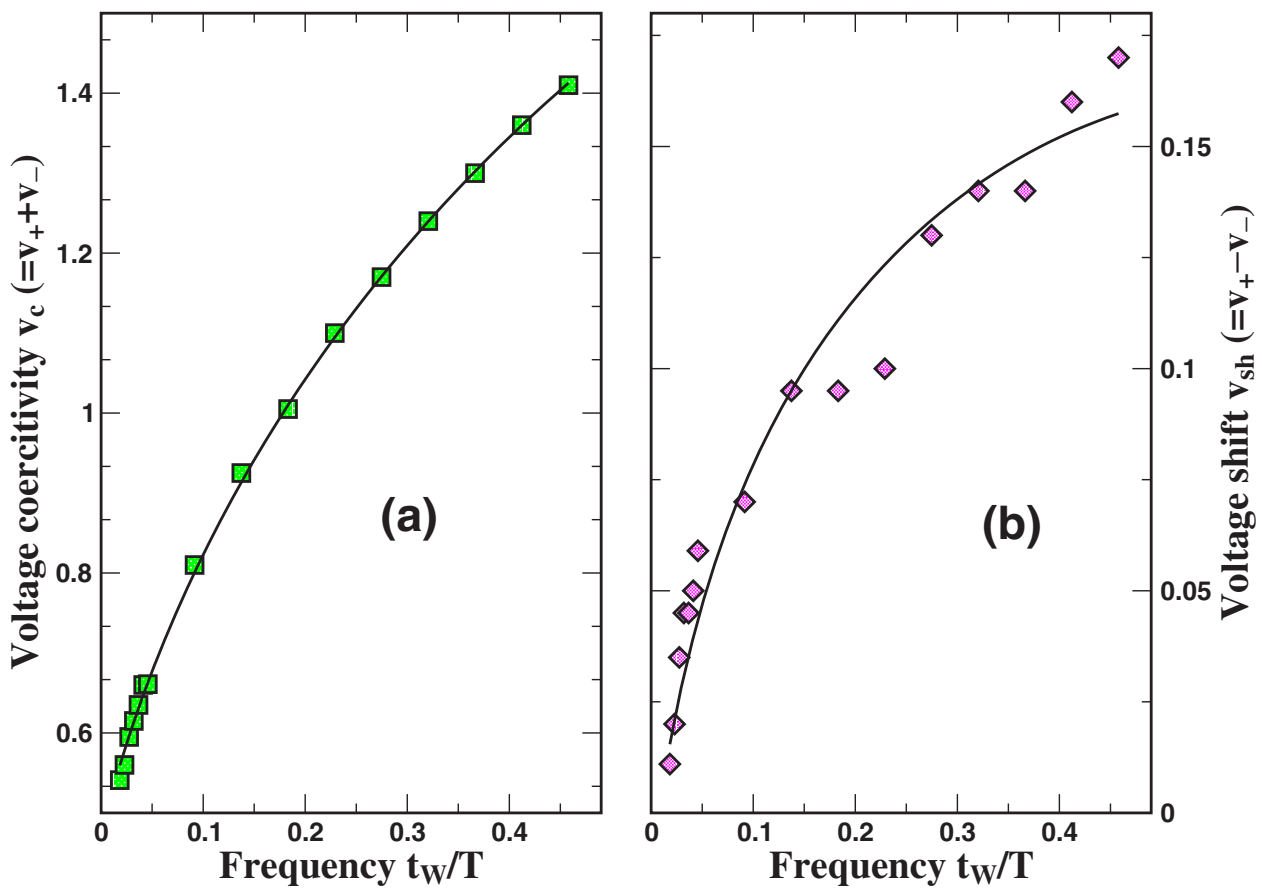

FIG. 9. (Color online) (a) Voltage coercitivity (squares) and (b) shift of the hysteresis loop (diamonds) measured in the FLC-497 cell as a function of frequency. Solid lines represent the results of modeling at $r_{e}=80$ and $w_{p}=-0.78$ using the approximate relations: $\left(u_{-}-1\right) f_{w}$ $\approx 0.15+4.7 f_{w}$ and $\left(u_{+}-1\right) f_{w} \approx 15.6-4.24 f_{w}$.

$$
\frac{\partial u}{\partial \tau} \equiv \dot{u}=-\frac{\partial U(u)}{\partial u}+r(\tau), \quad U(u)=U_{0}(u)-w_{p} u,
$$

where the anchoring energy potential $U_{0}$ is taken to be a piecewise quadratic function of the following form:

$$
U_{0}(u)= \begin{cases}\epsilon_{-} u^{2} / 2+\left(1+\epsilon_{-}\right)(u+1 / 2), & u<-1, \\ -u^{2} / 2, & -1 \leqslant u \leqslant 1, \\ \epsilon_{+} u^{2} / 2-\left(1+\epsilon_{+}\right)(u-1 / 2), & u>1 .\end{cases}
$$

Referring to Fig. 10, the effective potential $U(u)$ defined in Eqs. (76) and (77) represents a double-well potential with the two minima located at $u_{\min }^{(+)}=\left(1+\epsilon_{+}+w_{p}\right) / \epsilon_{+}$and $u_{\min }^{(-)}=-(1$ $\left.+\epsilon_{-}-w_{p}\right) / \epsilon_{-}$.

Clearly, our original model (37) can be regarded as the limiting case of the modified model (76) in which the parameters $\epsilon_{ \pm}$are equal to zero, $\epsilon_{ \pm}=0$. In this limit, the parameters $\left|u_{\min }^{( \pm)}\right|$become infinitely large and the minima shift at infinity in opposite directions.

The key results obtained in Ref. [39] for square wave voltage at $\epsilon_{ \pm}=\epsilon$ can be summarized as follows.

(a) There are two limiting frequencies, $f_{1}$ and $f_{2}$,

$$
f_{1,2} \approx 2^{-1} \epsilon r_{e}\left[1-\left|w_{p}\right|+2 \epsilon \mp \sqrt{\left(1-\left|w_{p}\right|\right)^{2}-4 \epsilon\left|w_{p}\right|}\right)^{-1},
$$

so that the cycles are unstable at $f_{2}<f_{w}<f_{1}$.

(b) Unstable cycles can only be formed if the separation between the minima of the effective potential is sufficiently large and $\epsilon<\epsilon_{c}$, where

$$
\boldsymbol{\epsilon}_{c} \approx\left|w_{p}\right|^{-1}\left(1-\left|w_{p}\right|\right)^{2}\left[2-\left(1+\left|w_{p}\right|\right)^{2} r_{e}^{-2}\right] / 8
$$

(c) When $\epsilon<\epsilon_{c}$, in addition to unstable cycles, there is the branch of stable cycles in the low frequency region below the critical frequency $f_{c}, f_{w}<f_{c}$ :
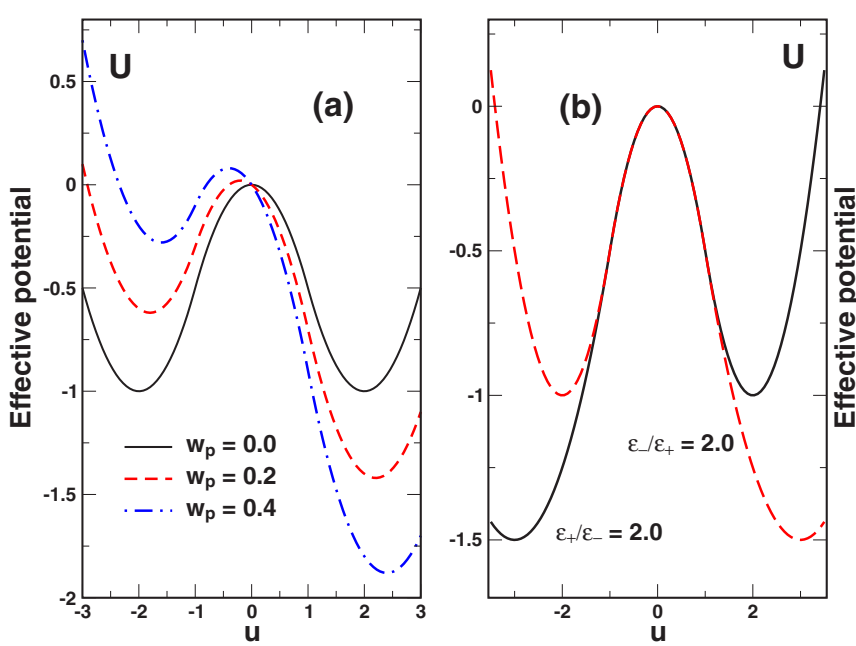

FIG. 10. (Color online) Double-well effective potential as a function of $u$. Two cases are shown: (a) $\epsilon_{ \pm}=1$ at various values of the polar anchoring parameter $w_{p}$; and (b) $\epsilon_{+} \neq \epsilon_{-}$at $w_{p}=0$. Minima and maximum of the potential are located at $u_{\min }^{( \pm)}= \pm(1$ $\left.+\epsilon_{ \pm} \pm w_{p}\right) / \epsilon_{ \pm}$and $u_{\max }=-w_{p}$, respectively. 


$$
f_{c} \approx 2^{-1} \epsilon r_{e}\left[1+\left|w_{p}\right|+2 \epsilon+\sqrt{\left(1+\left|w_{p}\right|\right)^{2}+4 \epsilon\left|w_{p}\right|}\right]^{-1} .
$$

(d) At $w_{p} \neq 0$, the low frequency region is separated from the frequency interval of unstable cycles by the gap of the width $f_{2}-f_{c}$.

These results suggest that the periodic regimes of switching are sensitive to the shape of the anchoring potential which plays an important part in the mechanism rendering the cycles unstable. In our case this is the Rapini-Papoular potential that predicts no stable cycles. Such instability, however, may imply that the potential fails to describe large deviations of the director from the easy axis in the course of the switching process. For similar reasons, modifications of the potential were considered in very recent studies on the dynamics of a pitch jump in cholesteric liquid crystal cells $[46,47]$.

Note that, similar to Eqs. (64) and (65), the expressions (78)-(80) are simplified so as to give the asymptotic formulas in the high voltage region with $r_{e} \geqslant 10$. For brevity we have dropped a detailed analysis and an extended discussion that can be found in Ref. [39].

The uniform theory cannot be directly applied to more complicated cases with spatially inhomogeneous orientational structures involved. The problems such as the soliton (kink) mechanism of switching [48-50] and dynamics in the chevron geometry $[51,52]$ generally require using a more comprehensive approach going beyond the scope of the uniform theory. Nevertheless, it is pertinent to note that, under certain circumstances, the switching dynamics of inhomogeneous structures can be effectively reduced to a uniform model for relevant spatially independent variables using a trial solution in the first approximation. So, we hope that the results of this paper will stimulate further progress in the field.

\section{ACKNOWLEDGMENTS}

This work was supported by CERG Grant No. 612406 and RGC Grants No. HKUST6149/04E and No. DAG05/ 06.EG14. The authors are thankful to Professor H.-S. Kwok for useful conversations.
[1] R. B. Meyer, L. Liebert, L. Strezlecki, and P. Keller, J. Phys. (France) Lett. 36, L69 (1975).

[2] Ferroelectric Liquid Crystals, edited by G. W. Taylor, Vol. 7 of Ferroelectricity and Related Phenomena (Gordon and Breach Science, New York, 1991).

[3] S. T. Lagerwall, Ferroelectric and Antiferroelectric Liquid Crystals (Wiley-VCH, New York, 1999).

[4] I. Muševič, R. Blinc, and B. Žekš, Physics of Ferroelectric and Antiferroelectric Liquid Crystals (World Scientific, Singapore, 2000).

[5] N. A. Clark and S. T. Lagerwall, Appl. Phys. Lett. 36, 899 (1980).

[6] M. A. Handschy and N. A. Clark, Appl. Phys. Lett. 41, 39 (1982).

[7] M. A. Handschy, N. A. Clark, and S. T. Lagerwall, Phys. Rev. Lett. 51, 471 (1983).

[8] M. A. Handschy and N. A. Clark, Ferroelectrics 59, 69 (1984).

[9] P. Schiller, Cryst. Res. Technol. 21, 301 (1986).

[10] I. Dahl, S. T. Lagerwall, and K. Skarp, Phys. Rev. A 36, 4380 (1983).

[11] T. Sako, N. Itoh, A. Sakaigawa, and M. Koden, Appl. Phys. Lett. 71, 461 (1997).

[12] V. Panov, J. K. Vij, and N. M. Shtykov, Liq. Cryst. 28, 615 (2001).

[13] M. Nakagawa, Jpn. J. Appl. Phys., Part 1 39, 4068 (2000).

[14] S. M. Said and S. J. Elston, Liq. Cryst. 28, 561 (2001).

[15] H. Pauwels and S. T. Lagerwall, Liq. Cryst. 28, 573 (2001).

[16] S. Essid, M. Manai, A. Gharbi, J. P. Marcerou, and J. P. Rouillon, Liq. Cryst. 32, 307 (2005).

[17] D. K. Arrowsmith and C. M. Place, Dynamical Systems. Differential Equations, Maps and Chaotic Behaviour (Chapman \& Hall, London, 1992).

[18] Y. A. Kuznetsov, Elements of Applied Bifurcation Theory, Vol. 112 of Applied Mathematical Sciences 2nd ed. (Springer, New
York, 1998).

[19] A. Rapini and M. Papoular, J. Phys. (Paris), Colloq. 30, 54 (1969).

[20] J. D. Parsons, Phys. Rev. Lett. 41, 877 (1978).

[21] W. E. McMullen, Phys. Rev. A 38, 6384 (1988).

[22] M. A. Osipov and T. J. Sluckin, J. Phys. II 3, 793 (1993).

[23] M. A. Osipov, T. J. Sluckin, and S. J. Cox, Phys. Rev. E 55, 464 (1997).

[24] N. A. Clark and S. T. Lagerwall, Ferroelectrics 59, 25 (1984).

[25] P. G. de Gennes and J. Prost, The Physics of Liquid Crystals (Clarendon Press, Oxford, 1993).

[26] I. Dahl and S. T. Lagerwall, Ferroelectrics 58, 215 (1984).

[27] N. Vaupotič and M. Čopič, Phys. Rev. E 68, 061705 (2003).

[28] J. Xue and N. A. Clark, Phys. Rev. Lett. 64, 307 (1990).

[29] R.-F. Shao, J. E. Maclennan, N. A. Clark, D. J. Dyer, and D. M. Walba, Liq. Cryst. 28, 117 (2001).

[30] E. P. Pozhidaev, V. G. Chigrinov, Y. P. Panarin, and V. P. Volflusev, Mol. Mater. 2, 225 (1993).

[31] N. J. Mottram, and S. J. Elston, Phys. Rev. E 62, 6787 (2000).

[32] M. Čopič, J. E. Maclennan, and N. A. Clark, Phys. Rev. E 65, 021708 (2002).

[33] M. J. O'Callaghan, Phys. Rev. E 67, 011710 (2003).

[34] L. M. Blinov, S. P. Palto, E. P. Pozhidaev, Y. P. Bobylev, V. M. Shoshin, A. L. Andreev, F. V. Podgornov, and W. Haase, Phys. Rev. E 71, 051715 (2005).

[35] L. M. Blinov, E. P. Pozhidaev, F. V. Podgornov, S. A. Pikin, S. P. Palto, A. Sinha, A. Yasuda, S. Hashimoto, and W. Haase, Phys. Rev. E 66, 021701 (2002).

[36] N. A. Clark and S. T. Lagerwall, in Ferroelectric Liquid Crystals, edited by G. W. Taylor Vol. 7 of Ferroelectricity and Related Phenomena, (Gordon and Breach Science, New York, 1991), Chap. 1, pp. 1-97.

[37] C. Reynaerts and A. D. Vos, J. Phys. D 22, 1504 (1989).

[38] C. Reynaerts and A. D. Vos, Ferroelectrics 113, 439 (1991). 
[39] A. D. Kiselev, V. G. Chigrinov, and E. P. Pozhidaev, e-print arXiv:cond-mat/0609302, URL http://www.arXiv.org.

[40] V. Chigrinov, E. Prudnikova, V. Kozenkov, H. Kwok, H. Akiyama, T. Kawara, H. Takada, and H. Takatsu, Liq. Cryst. 29, 1321 (2002).

[41] A. Z. Rabinovich, M. V. Loseva, N. I. Chernova, E. P. Pozhidaev, O. S. Petrashevich, and J. S. Narkevich, Liq. Cryst. 6, 533 (1989).

[42] V. Chigrinov, A. Muravski, and H. S. Kwok, Phys. Rev. E 68, 061702 (2003).

[43] A. D. Kiselev, V. G. Chigrinov, and D. D. Huang, Phys. Rev. E 72, 061703 (2005).

[44] A. Murauski, V. Chigrinov, A. Muravsky, Fion Sze-Yan Yeung, J. Ho, and H.-S. Kwok, Phys. Rev. E 71, 061707 (2005).

[45] D. C. Ulrich, M. J. Cherrill, and S. J. Elston, Liq. Cryst. 23,
797 (1997).

[46] V. A. Belyakov, I. W. Stewart, and M. A. Osipov, Phys. Rev. E 71, 051708 (2005).

[47] V. A. Belyakov, M. A. Osipov, and I. W. Stewart, J. Phys.: Condens. Matter 18, 4443 (2006).

[48] I. Abdulhalim, G. Moddel, and N. A. Clark, J. Appl. Phys. 67, 820 (1994).

[49] E. I. Demikhov, S. A. Pikin, and E. S. Pikina, Phys. Rev. E 52, 6250 (1995).

[50] E. N. Tsoy, I. W. Stewart, and F. K. Abdullaev, Phys. Rev. E 60, 5568 (1999).

[51] N. Vaupotič, V. Grubelnik, and M. Čopič, Phys. Rev. E 62, 2317 (2000).

[52] L. D. Hazelwood and T. J. Sluckin, Liq. Cryst. 31, 683 (2004). 